\title{
主
}

\section{Sobolev-type orthogonal polynomials on the unit circle}

\author{
F. Marcellán ${ }^{a, *}$, L. Moral $^{b}$ \\ a Departamento de Matemáticas, Universidad Carlos III de Madrid, Calle Butarque 15, \\ 28911 Leganés Madrid, Spain \\ ${ }^{\mathrm{b}}$ Departamento de Matemática Aplicada, Universidad de Zaragoza, Zaragoza, Spain
}

\begin{abstract}
This paper deals with polynomials orthogonal with respect to a Sobolev type inner product

$$
\langle f, g\rangle=\int_{-\pi}^{\pi} f\left(\mathrm{e}^{\mathrm{i} \theta}\right) \overline{g\left(\mathrm{e}^{\mathrm{i} \theta}\right)} \mathrm{d} \mu\left(\mathrm{e}^{\mathrm{i} \theta}\right)+\mathbf{f}(c) A[\mathbf{g}(c)]^{H},
$$

where $\mu$ is a positive Borel measure supported on $[\pi, \pi), A$ is a nonsingular matrix and $|c|>1$. We denote $\mathbf{f}(c)=\left(f(c), f^{\prime}(c), \ldots, f^{(p)}(c)\right)$ and $\mathbf{v}^{H}$ the transposed conjugate of the vector $\mathbf{v}$. We establish the connection of such polynomials with orthogonal poly nomials on the unit circle with respect to the measure $\mathrm{d} v(z)=|z \quad c|^{2 p+2} \mathrm{~d} \mu(z)$ $\left(z=\mathrm{e}^{\mathrm{i} \theta}, p \in \mathbb{N}\right)$. Finally, we deduce the relative asymptotics for both families of or thogonal polynomials.
\end{abstract}

Keywords: Orthogonal polynomials; Reflection parameters; Nevai class; Sobolev inner products

\section{Introduction}

In the last years the analysis of polynomials orthogonal with respect to a Sobolev inner product

$$
\langle f, g\rangle=\int_{E} f(z) \overline{g(z)} \mathrm{d} \mu(z)+\sum_{i}^{p} \lambda_{i} \int_{E_{i}} f^{(i)}(z) \overline{g^{(i)}(z)} \mathrm{d} \mu_{i}(z)
$$

${ }^{*}$ Corresponding author.

E mail addresses: pacomarc@ing.uc3m.es (F. Marcellán), 1moral@posta.unizar.es (L. Moral). 
has attracted the interest of several people. In fact, the comparison with the very well-known properties of the standard case $\left(\lambda_{i}=0\right)$ when $E=E_{1}=\cdots=E_{p}$ is the real line or the unit circle was one of the most important challenges.

In the first situation, a basic effort was put on the asymptotic behavior of the new family with respect to the standard one (see [10] as well as [9]). The improvement of the relative asymptotics in the most general situation (when in the nonstandard part appear mixed derivatives) leads to significant results strongly connected with rational approximation and the estimates of the speed of convergence.

More recently [1] was started an analog study when $p=1, \mathrm{~d} \mu_{1}$ is an atomic measure concentrated in a point $c$ outside the unit circle $\mathbb{T}$, and $E=E_{1}=\mathbb{T}$. Later on, in [7] an extension of the above situation is considered when the nonstandard component is

$$
\left(f^{\prime}\left(c_{1}\right), f^{\prime}\left(c_{2}\right), \ldots, f^{\prime}\left(c_{k}\right)\right) A\left[\left(g^{\prime}\left(c_{1}\right), g^{\prime}\left(c_{2}\right), \ldots, g^{\prime}\left(c_{k}\right)\right)\right]^{H},
$$

and $\left|c_{i}\right|>1, i=1,2, \ldots, k$.

The aim of our present contribution is to study in a second step the algebraic properties as well as the asymptotic behavior of orthogonal polynomials when the inner product is

$$
\begin{aligned}
\langle f, g\rangle=\int_{-\pi}^{\pi} f\left(\mathrm{e}^{\mathrm{i} \theta}\right) \overline{g\left(\mathrm{e}^{\mathrm{i} \theta}\right)} \mathrm{d} \mu\left(\mathrm{e}^{\mathrm{i} \theta}\right) & \\
& \quad+\left(f(c), f^{\prime}(c), \ldots, f^{(p)}(c)\right) A\left[\left(g(c), g^{\prime}(c), \ldots, g^{(p)}(c)\right)\right]^{H}
\end{aligned}
$$

and $A$ is a nonsingular matrix.

The structure of the paper is the following. In Section 2 we introduce the basic tools about the measures $\mu$ to be considered. They belong to the so-called Nevai Blumenthal class [12] and, in such a case, the asymptotic behavior of the corresponding orthogonal polynomials is well known. In Section 3 we analyze a polynomial perturbation of such measures and we prove that they belong to the Nevai Blumenthal class. Furthermore, we obtain explicit expressions for the sequences of orthogonal polynomials associated with them as well as their asymptotic behavior. Section 4 is focussed in the analysis of the behavior of polynomials orthonormal with respect to (1.1) and their dependence of $A$. In particular, we emphasize the case when $A$ is a positive definite matrix. Finally, in Section 5 we obtain the limit of the ratio of the leading coefficients for both orthonormal sequences when $A$ is nonsingular, as well as the relative asymptotics for them.

A third step, when several points are considered as well as $A$ is a positive semidefinite matrix has been very recently obtained in [2]. Notice that, in our case, the inner product is not Hermitian at all but we can give necessary and sufficient conditions for the existence of (left) orthogonal polynomials even 
when $A$ is a singular matrix. Even more, our approach is different in terms of the techniques that we have developed here.

\section{Basic tools}

We shall denote by $\mathbb{N}$ the set of all nonnegative integer numbers. Let $\mathbb{P}$ be the vector space of polynomials with complex coefficients, and $\mathbb{P}_{n}$ the subspace of the polynomials whose degree is less than or equal to $n$.

Let $\mu$ be a probability measure supported on the unit circle $\mathbb{T}=\{z \in \mathbb{C}:|z|=1\}$. Let us define on $\mathbb{P}$ the inner product

$$
(f, g):=\int_{|z|} f(z) \overline{g(z)} \mathrm{d} \mu(z), \quad f, g \in \mathbb{P} .
$$

Then, we have an uniquely determined sequence of orthonormal polynomials $\left(\varphi_{n}\right)_{n \in \mathbb{N}}$

$$
\begin{aligned}
& \varphi_{0}(z)=1 ; \quad \varphi_{n}(z):=\kappa_{n} z^{n}+(\text { lower degree terms }), \quad \kappa_{n}>0, \\
& \left(\varphi_{m}, \varphi_{n}\right)=\delta_{m n}, \quad m, n \in \mathbb{N} .
\end{aligned}
$$

It is well known (see $[3,13,14]$ ) that the polynomial sequence $\left(\varphi_{n}\right)_{n \in \mathbb{N}}$ satisfies the following recurrence relation

$$
\kappa_{n} \varphi_{n+1}(z)=\kappa_{n+1} z \varphi_{n}(z)+\varphi_{n+1}(0) \varphi_{n}^{*}(z), \quad n \in \mathbb{N},
$$

where $\varphi_{n}^{*}(z)=z^{n} \overline{\varphi_{n}}(1 / z)$ is the reversed polynomial of $\varphi_{n}(z)$, as well as, for $n \geqslant 1$,

(i) The zeros of the polynomial $\varphi_{n}(z)$ lie on $|z|<1$,

(ii) $\left|\varphi_{n}(0) / \kappa_{n}\right|<1$.

The $n$th kernel polynomial $K_{n}(z, y)$ is defined by

$$
K_{n}(z, y):=\sum_{k}^{n} \varphi_{k}(z) \overline{\varphi_{k}(y)}, \quad n \in \mathbb{N}, \quad K_{-1}(z, y)=0 .
$$

They satisfy the reproducing property

$$
\left(f(z), K_{n}(z, y)\right)=f(y), \quad f \in \mathbb{P}_{n},
$$

as well as the Christoffel Darboux formula (see $[4,6,14]$ )

$$
(1-\bar{y} z) K_{n-1}(z, y)=\varphi_{n}^{*}(z) \overline{\varphi_{n}^{*}(y)}-\varphi_{n}(z) \overline{\varphi_{n}(y)}, \quad n \in \mathbb{N} .
$$

If we define

$$
K_{n}^{(i, j)}(z, y):=\frac{\partial^{i+j}}{\partial^{i} z \partial^{j} \bar{y}} K_{n}(z, y)=\sum_{k 0}^{n} \varphi_{k}^{(i)}(z) \overline{\varphi_{k}^{(j)}(y)},
$$


then, for all $f \in \mathbb{P}_{n}$,

$$
\left(f(z), K_{n}^{(0, j)}(z, y)\right)=f^{(j)}(y)
$$

holds.

On the other hand, we have

Proposition 2.1. Let $c \in \mathbb{C}, q \in \mathbb{N}$ and $M(n) \in \mathbb{C}^{(q+1, q+1)}$ be the matrix

$$
M(n):=\left(\begin{array}{cccc}
K_{n}(c, c) & K_{n}^{(1,0)}(c, c) & \ldots & K_{n}^{(q, 0)}(c, c) \\
K_{n}^{(0,1)}(c, c) & K_{n}^{(1,1)}(c, c) & \ldots & K_{n}^{(q, 1)}(c, c) \\
\vdots & \vdots & \vdots & \vdots \\
K_{n}^{(0, q)}(c, c) & K_{n}^{(1, q)}(c, c) & \ldots & K_{n}^{(q, q)}(c, c)
\end{array}\right) .
$$

Then, $M(n)$ is positive definite for $n \geqslant q$.

Proof. Obviously, $M(n)$ is Hermitian. Let $A(n) \in \mathbb{C}^{(q+1, n+1)}$ be the matrix

$$
\begin{aligned}
A(n) & =\left(\begin{array}{cccc}
\varphi_{0}(c) & \varphi_{1}(c) & \ldots & \varphi_{n}(c) \\
\varphi_{0}^{\prime}(c) & \varphi_{1}^{\prime}(c) & \ldots & \varphi_{n}^{\prime}(c) \\
\vdots & \vdots & \vdots & \vdots \\
\varphi_{0}^{(q)}(c) & \varphi_{1}^{(q)}(c) & \ldots & \varphi_{n}^{(q)}(c)
\end{array}\right) \\
& =\left(\begin{array}{ccccc}
\kappa_{0} & \cdots & \cdots & \ldots & \cdots \\
0 & \kappa_{1} & \cdots & \cdots & \cdots \\
\vdots & \vdots & \vdots & \vdots & \vdots \\
0 & 0 & \cdots & q ! \kappa_{q} & \cdots
\end{array}\right)
\end{aligned}
$$

Because $\kappa_{j}>0$, then $\operatorname{rank} A(n)=\min \{q+1, n+1\}$. Furthermore,

$$
M(n)=A(n)[A(n)]^{H} .
$$

(Throughout the paper, $C^{H}$ denotes the transposed conjugate of the matrix $C$.) Then,

$$
\mathbf{x} M(n) \mathbf{x}^{H}=[\mathbf{x} A(n)][\mathbf{x} A(n)]^{H} \geqslant 0,
$$

for all $\mathbf{x} \in \mathbb{C}^{q+1}$, that is to say, $M(n)$ is, in general, a positive semidefinite matrix. For $n \geqslant q, \operatorname{rank} A(n)=q+1$ holds; thus, $\mathbf{x} M(n) \mathbf{x}^{H}=0$ iff $\mathbf{x}=0$.

Remark. The nonsingularity of the matrix $M(n)(n \geqslant q)$ is essential in the next sections. This fact can be assured when the sequence of orthogonal polynomials is associated with an inner product, i.e., a positive measure supported on $\mathbb{T}$. So, $M(n)$ is positive definite for $n \geqslant q$. 
Definition. (See $[11,12])$ Let $\mu$ be a probability measure supported on $\mathbb{T}$. We say that $\mu$ belongs to the Nevai Blumenthal class (in short, $\mathcal{N}$ class), if any of the following equivalent statements hold

(i) $\lim _{n \rightarrow \infty}\left(\kappa_{n+1} / \kappa_{n}\right)=1$,

(ii) $\lim _{n \rightarrow \infty}\left(\varphi_{n}(0) / \kappa_{n}\right)=0$,

(iii) $\lim _{n \rightarrow \infty}\left(\varphi_{n+1}(z) / \varphi_{n}(z)\right)=z$, uniformly in compact subsets of $|z| \geqslant 1$.

Furthermore, if $\mu \in \mathscr{N}$, then

$$
\lim _{n}\left(1 / \varphi_{n}(z)\right)=0, \quad \lim _{n}\left(\varphi_{n}^{*}(z) / \varphi_{n}(z)\right)=0,
$$

uniformly in compact subsets of $|z|>1$ (see [7,8]).

When the limit function is continuous, the uniform convergence in compact subsets is equivalent to locally uniform convergence. So, we write 1.u. for uniform convergence in compact subsets, because all the limit functions will be continuous.

Lemma 2.2. Assume that $p(z)=\prod_{j 1}^{n}\left(z-z_{j}\right)$, with $\left|z_{j}\right| \leqslant R$, and $H$ an arbitrary compact subset in $|z|>R$. Then, there are two positive real numbers $d=d(H)$ and $e=e(H)$ such that

$$
0<d \leqslant\left|\frac{p^{\prime}(z)}{n p(z)}\right| \leqslant e,
$$

for $z \in H$ (see [7]).

Proof. Denote $c=\min _{z \in H}\{|z|\}, C=\max _{z \in H}\{|z|\}$. Then, $R<c \leqslant C$. Since

$$
\frac{p^{\prime}(z)}{p(z)}=\sum_{j 1}^{n} \frac{1}{z-z_{j}}=\sum_{j 1}^{n} \frac{\bar{z}-\overline{z_{j}}}{\left|z-z_{j}\right|^{2}}
$$

we have

$$
\left|\frac{p^{\prime}(z)}{p(z)}\right|=\left|\bar{z} \sum_{j 1}^{n} \frac{1}{\left|z-z_{j}\right|^{2}}-\sum_{j 1}^{n} \frac{\overline{z_{j}}}{\left|z-z_{j}\right|^{2}}\right|=A\left|z-\sum_{j 1}^{n} \frac{\alpha_{j}}{A} z_{j}\right|,
$$

for all $z \in H$, where $\alpha_{j}=\left|z-z_{j}\right|^{-2}$, and $A=\sum \alpha_{j}$. The convex hull of $\left(z_{j}\right)_{j 1}^{n}$ lies on the disk $|z| \leqslant R$. Thus the second factor is bounded from below by $|z|-R$ and, therefore, by $c-R$. On the other hand, from $A \geqslant n \cdot \min \left\{\alpha_{j}\right\} \geqslant$ $n /(C+R)^{2}$,

$$
\left|\frac{p^{\prime}(z)}{p(z)}\right| \geqslant n \frac{c-R}{(C+R)^{2}}=n d
$$


follows. Besides,

$$
\left|\frac{p^{\prime}(z)}{p(z)}\right| \leqslant \sum_{j}^{n} \frac{|z|+\left|z_{j}\right|}{\left|z-z_{j}\right|^{2}} \leqslant \sum_{j}^{n} \frac{C+R}{(c-R)^{2}}=n \frac{C+R}{(c-R)^{2}}=n e .
$$

Corollary 2.3. In the conditions of the above lemma, the zeros of $p^{\prime}(z)$ lie on $|z| \leqslant R$.

Often we are going to use the following lemmas (see [7,12]).

Lemma 2.4. Let $P, Q$ be two polynomials in $\mathbb{P}$ whose degrees are not less than $n$. Then

$$
\frac{P^{(k)}(z)}{Q^{(k)}(z)}=\frac{Q^{(k-1)}(z)}{Q^{(k)}(z)}\left(\frac{P^{(k-1)}(z)}{Q^{(k-1)}(z)}\right)^{\prime}+\frac{P^{(k-1)}(z)}{Q^{(k-1)}(z)} .
$$

holds for $k=0,1, \ldots, n$.

Lemma 2.5. If $\mu \in \mathscr{N}$ and $k \in \mathbb{N}$, then

(i) $\lim _{n}\left(\varphi_{n}^{(k)}(z) /\left(\varphi_{n}^{(k+1)}(z)\right)\right)=0$,

(ii) $\lim _{n}\left(\varphi_{n+1}^{(k)}(z) / \varphi_{n}^{(k)}(z)\right)=z$,

(iii) $\lim _{n}\left(1 / \varphi_{n}^{(k)}(z)\right)=0$,

l. u. in $|z|>1$.

Lemma 2.6. If $\mu \in \mathscr{N}$, then the following statements are fulfilled

(i) For $i, j \in \mathbb{N}$,

$$
\lim _{n} \frac{K_{n}(i, j)}{\varphi_{z}(i) \overline{\varphi_{z}(j)}} y=\frac{1}{z \bar{y}-1}
$$

l. u. in $\{|z|>1\} \times\{|y|>1\}$. In particular, for $|c|>1$ we have

$$
\lim _{n} \frac{\varphi_{n}^{(i)}(c) \overline{\varphi_{n}^{(j)}(c)}}{K_{n-1}^{(i, j)}(c, c)}=|c|^{2}-1,
$$

(ii) $\lim _{n}\left(K_{n-1}(z, c) / \varphi_{n}(z) \overline{\varphi_{n}(c)}\right)=(1 / \bar{c} z-1)$ l. u. in $|z|>1$ if $|c| \geqslant 1$ and uniformly in $|z| \geqslant 1$ if $|c|>1$,

(iii) If $|c|=1$, then $\lim _{n}\left(\left|\varphi_{n}(c)\right|^{2} / K_{n-1}(c, c)\right)=0$.

\section{Modification $|z-c|^{2(q+1)} \mathrm{d} \mu$ of the measure $\mu$}

We will try to analyze if there is a polynomial $\omega_{n}$ such that a relation

$$
(z-c)^{q+1} \omega_{n}(z)=\alpha_{n}\left[\varphi_{n+q+1}(z)+\sum_{i}^{q} \beta_{i n} K_{n+q}^{(0, i)}(z, c)\right], \quad n \in \mathbb{N}
$$


holds, where $c \in \mathbb{C}, q \in \mathbb{N}, \alpha_{n} \neq 0$ for all $n$, and $\left(\varphi_{n}\right)_{n \in \mathbb{N}}$ is the orthogonal polynomial sequence related to a probability measure $\mu$ supported on $\mathbb{T}$.

Let us write

$$
\begin{aligned}
& \boldsymbol{\beta}_{n}=\left[\beta_{0 n}, \beta_{1 n}, \ldots, \beta_{q n}\right], \\
& \mathbf{K}_{n+q}(z, c)=\left[K_{n+q}(z, c), K_{n+q}^{(0,1)}(z, c), \ldots, K_{n+q}^{(0, q)}(z, c)\right] .
\end{aligned}
$$

Then, the above relation can be expressed as

$$
(z-c)^{q+1} \omega_{n}(z)=\alpha_{n}\left[\varphi_{n+q+1}(z)+\boldsymbol{\beta}_{n}\left[\mathbf{K}_{n+q}(z, c)\right]^{\mathrm{T}}\right] .
$$

Lemma 3.1. Let $n \geqslant q+1$. Then

$$
1+\boldsymbol{\varphi}_{n} c[M(n-1)]^{-1}\left[\boldsymbol{\varphi}_{n}(c)\right]^{H}=\frac{\operatorname{det}[M(n)]}{\operatorname{det}[M(n-1)]}>0
$$

holds, with $\boldsymbol{\varphi}_{n}(c)=\left[\varphi_{n}(c), \varphi_{n}^{\prime}(c), \ldots, \varphi_{n}^{(q)}(c)\right]$.

Proof. Let us consider the matrix identities

$$
\begin{aligned}
& \left(\begin{array}{ll}
I & {\left[\boldsymbol{\varphi}_{n}(c)\right]^{H}} \\
0 & 1
\end{array}\right)\left(\begin{array}{cc}
M(n-1) & -\left[\boldsymbol{\varphi}_{n}(c)\right]^{H} \\
\boldsymbol{\varphi}_{n}(c) & 1
\end{array}\right) \\
& =\left(\begin{array}{cc}
M(n) & 0 \\
\boldsymbol{\varphi}_{n}(c) & 1
\end{array}\right),\left(\begin{array}{cc}
M(n-1) & -\left[\boldsymbol{\varphi}_{n}(c)\right]^{H} \\
\boldsymbol{\varphi}_{n}(c) & 1
\end{array}\right)\left(\begin{array}{cc}
I & {[M(n-1)]^{-1}\left[\boldsymbol{\varphi}_{n}(c)\right]^{H}} \\
0 & 1
\end{array}\right) \\
& =\left(\begin{array}{cc}
M(n-1) & 0 \\
\boldsymbol{\varphi}_{n}(c) & 1+\boldsymbol{\varphi}_{n}(c)[M(n-1)]^{-1}\left[\boldsymbol{\varphi}_{n}(c)\right]^{H}
\end{array}\right) .
\end{aligned}
$$

The determinants of both left-hand side expressions are equal. For the righthand side expressions, we get

$$
\operatorname{det}[M(n)]=\operatorname{det}[M(n-1)]\left(1+\boldsymbol{\varphi}_{n}(c)[M(n-1)]^{-1}\left[\boldsymbol{\varphi}_{n}(c)\right]^{H}\right) .
$$

Thus, from Proposition 2.1, the positivity of $1+\boldsymbol{\varphi}_{n}(c)[M(n-1)]^{-1}\left[\boldsymbol{\varphi}_{n}(c)\right]^{H}$ follows.

Theorem 3.2. There is one and only one polynomial sequence $\left(\omega_{n}\right)_{n \in \mathbb{N}}$ verifying (3.1) (up to a non-zero factor for each $n$ ), which is orthogonal with respect to the inner product

$$
\begin{aligned}
(f, g)_{q+1} & =\left((z-c)^{q+1} f(z),(z-c)^{q+1} g(z)\right) \\
& =\int_{|z| 1} f(z) \overline{g(z)}|z-c|^{2(q+1)} \mathrm{d} \mu(z) .
\end{aligned}
$$


[Notice that $\mathrm{d} \mu_{q+1}=|z-c|^{2(q+1)} \mathrm{d} \mu$ is also a positive Borel measure supported on $\mathbb{T}$.]

Proof. The necessary and sufficient condition for the existence of $\omega_{n}$ is

$$
0=\alpha_{n}\left[\varphi_{n+q+1}^{(j)}(c)+\sum_{i}^{q} \beta_{i n} K_{n+q}^{(j, i)}(c, c)\right], \quad j=0, \ldots, q,
$$

or

$$
\boldsymbol{\beta}_{n} M(n+q)=-\boldsymbol{\varphi}_{n+q+1}(c),
$$

which is obtained by derivation of (3.1) up to $(q+1)$ th order, and evaluating it at $z=c$.

Thus, the positive definiteness for $M(n+q)$ implies that

$$
\boldsymbol{\beta}_{n}=-\boldsymbol{\varphi}_{n+q+1}(c)[M(n+q)]^{-1}
$$

is the only solution which yields a polynomial solution.

It is obvious that deg $\omega_{n}=n$. Also, for $k=0,1, \ldots, n-1$, we have

$$
\begin{aligned}
&\left(\omega_{n}(z), z^{k}\right)_{q+1}=\alpha_{n}\left[\left(\varphi_{n+q+1}(z),(z-c)^{q+1} z^{k}\right)\right. \\
&\left.+\sum_{i 0}^{q} \beta_{i n}\left(K_{n+q}^{(0, i)}(z, c),(z-c)^{q+1} z^{k}\right)\right] \\
&=\left.\alpha_{n} \sum_{i 0}^{q} \beta_{i n} \frac{\mathrm{d}^{i}}{\mathrm{~d} z^{i}}\left[(z-c)^{q+1} z^{k}\right]\right|_{z c}=0 .
\end{aligned}
$$

On the other hand,

$$
\begin{aligned}
\left(\omega_{n}, \omega_{n}\right)_{q+1}=\left|\alpha_{n}\right|^{2}\left[\left(\varphi_{n+q+1}(z), \varphi_{n+q+1}(z)\right)\right. \\
\left.\quad+\sum_{i, j 0}^{q}\left(\beta_{i n} K_{n+q}^{(0, i)}(z, c), \beta_{j n} K_{n+q}^{(0, j)}(z, c)\right)\right] \\
=\left|\alpha_{n}\right|^{2}\left(1+\boldsymbol{\beta}_{n} M(n+q) \boldsymbol{\beta}_{n}^{H}\right) \\
=\left|\alpha_{n}\right|^{2}\left(1+\boldsymbol{\varphi}_{n+q+1}(c)[M(n+q)]\left[\boldsymbol{\varphi}_{n+q+1}(c)\right]^{H}\right) \\
=\left|\alpha_{n}\right|^{2} \frac{\operatorname{det}[M(n+q+1)]}{\operatorname{det}[M(n+q)]},
\end{aligned}
$$

which is positive, according to Lemma 3.1. 
An alternative proof appears in [5].

The polynomials $\omega_{n}$ can be chosen orthonormal, with a suitable $\alpha_{n} \neq 0$, in the following way. Assume that

$$
\begin{aligned}
& \omega_{n}(z)=\eta_{n} z^{n}+(\text { lower degree terms }), \quad n \in \mathbb{N}, \\
& \left(\omega_{n}, \omega_{n}\right)_{q+1}=1 .
\end{aligned}
$$

Since $\alpha_{n}=\eta_{n} /\left(\kappa_{n+q+1}\right)$, we have

$$
\kappa_{n+q+1}=\eta_{n}\left(1+\boldsymbol{\varphi}_{n+q+1}(c)[M(n+q)]^{-1}\left[\boldsymbol{\varphi}_{n+q+1}(c)\right]^{H}\right)^{1 / 2},
$$

and, thus, (3.1) becomes

$$
(z-c)^{q+1} \omega_{n}(z)=\alpha_{n}\left(\varphi_{n+q+1}(z)-\boldsymbol{\varphi}_{n+q+1}(c)[M(n+q)]^{-1}\left[\mathbf{K}_{n+q}(z, c)\right]^{\mathrm{T}}\right)
$$

for the orthonormal polynomial sequence $\left(\omega_{n}\right)_{n \in \mathbb{N}}$.

We can obtain a recursive expression of the $\alpha_{n}$ coefficients in the following way. Let $\varphi_{n}\left(z ; \mathrm{d} \mu_{j+1}\right):=\omega_{n}(z)$ be the $n$th orthonormal polynomial defined by (3.3) for $j=0, \ldots, q$, and $K_{n}\left(z, y ; \mathrm{d} \mu_{j}\right)$ the corresponding $n$th kernel polynomial. Write

$$
\kappa_{n}\left(\mathrm{~d} \mu_{j+1}\right)=\eta_{n} .
$$

Then, we have

$$
\begin{aligned}
& (z-c) \varphi_{n}\left(z ; \mathrm{d} \mu_{j+1}\right)=\alpha_{n}^{(j)}\left[\varphi_{n+1}\left(z ; \mathrm{d} \mu_{j}\right)-\beta_{n}^{(j)} K_{n}\left(z, c ; \mathrm{d} \mu_{j}\right)\right], \\
& \quad j=0,1, \ldots, q,
\end{aligned}
$$

with $\varphi_{n}\left(z ; \mathrm{d} \mu_{0}\right)=\varphi_{n}(z), K_{n}\left(z, c ; \mathrm{d} \mu_{0}\right)=K_{n}(z, c)$. Thus,

$$
\alpha_{n}^{(j)}=\frac{\kappa_{n}\left(\mathrm{~d} \mu_{j+1}\right)}{\kappa_{n+1}\left(\mathrm{~d} \mu_{j}\right)}=\left[1+\frac{\left|\varphi_{n+1}\left(c ; \mathrm{d} \mu_{j}\right)\right|^{2}}{K_{n}\left(c, c ; \mathrm{d} \mu_{j}\right)}\right]^{-1 / 2}
$$

and

$$
\beta_{n}^{(j)}=\frac{\varphi_{n+1}\left(c ; \mathrm{d} \mu_{j}\right)}{K_{n}\left(c, c ; \mathrm{d} \mu_{j}\right)}
$$

follow with

$$
\alpha_{n}=\prod_{j}^{q} \alpha_{n+q-j}^{(j)}=\prod_{j}^{q}\left[1+\frac{\left|\varphi_{n+q-j+1}\left(c ; \mathrm{d} \mu_{j}\right)\right|^{2}}{K_{n+q-j}\left(c, c ; \mathrm{d} \mu_{j}\right)}\right]^{-1 / 2} .
$$


Lemma 3.3. Assume that $\mu \in \mathscr{N}$ and $|c| \geqslant 1$. Then, $\mu_{1} \in \mathscr{N}$, where $\mu_{1}$ is the measure $\mathrm{d} \mu_{1}=|z-c|^{2} \mathrm{~d} \mu$.

Proof. Since (3.4), we have

$$
\frac{(z-c) \varphi_{n}\left(z ; \mathrm{d} \mu_{1}\right)}{\kappa_{n}\left(\mathrm{~d} \mu_{1}\right)}=\frac{1}{\kappa_{n+1}}\left[\varphi_{n+1}(z)-\frac{\varphi_{n+1}(c)}{K_{n}(c, c)} K_{n}(z, c)\right],
$$

and, for $z=0$,

$$
-c \frac{\varphi_{n}\left(0 ; \mathrm{d} \mu_{1}\right)}{\kappa_{n}\left(\mathrm{~d} \mu_{1}\right)}=\frac{\varphi_{n+1}(0)}{\kappa_{n+1}}-\frac{\varphi_{n+1}(c)}{K_{n}(c, c)} \frac{K_{n}(0, c)}{\kappa_{n+1}} .
$$

Notice that, from the Christoffel Darboux formula (2.1)

$$
K_{n}(0, c)=\varphi_{n+1}^{*}(0) \overline{\varphi_{n+1}^{*}(c)}-\varphi_{n+1}(0) \overline{\varphi_{n+1}(c)} .
$$

Now,

$$
\begin{aligned}
-c \frac{\varphi_{n}\left(0 ; \mathrm{d} \mu_{1}\right)}{\kappa_{n}\left(\mathrm{~d} \mu_{1}\right)} & =\frac{\varphi_{n+1}(0)}{\kappa_{n+1}}-\frac{\varphi_{n+1}(c) \overline{\varphi_{n+1}^{*}(c)}}{K_{n}(c, c)}+\frac{\varphi_{n+1}(0)}{\kappa_{n+1}} \frac{\left|\varphi_{n+1}(c)\right|^{2}}{K_{n}(c, c)} \\
& =\frac{\varphi_{n+1}(0)}{\kappa_{n+1}}-\frac{\left|\varphi_{n+1}(c)\right|^{2}}{K_{n}(c, c)} \frac{\overline{\varphi_{n+1}^{*}(c)}}{\overline{\varphi_{n+1}(c)}}+\frac{\varphi_{n+1}(0)}{\kappa_{n+1}} \frac{\left|\varphi_{n+1}(c)\right|^{2}}{K_{n}(c, c)}
\end{aligned}
$$

because $\varphi_{n+1}^{*}(0)=\kappa_{n+1}$. From Lemma 2.6 and our hypothesis,

$$
\lim _{n} \frac{\varphi_{n}\left(0 ; \mathrm{d} \mu_{1}\right)}{\kappa_{n}\left(\mathrm{~d} \mu_{1}\right)}=0
$$

follows.

Lemma 3.4. Suppose that $\mu \in \mathscr{N}$. Then,

$$
\lim _{n} \frac{\varphi_{n}^{(j)}(z)}{\left[\varphi_{n}(z)\right]^{r+1}}=0 \quad \text { l. u. in }|z|>1
$$

holds, for $r>0$ and $j \in \mathbb{N}$.

Proof. If $j=0$, then let us consider $1 /\left[\varphi_{n}(z)\right]^{r}$, which tends to zero $l$. $u$. in $|z|>1$, because (2.2). Taking derivatives in the above expression, we get $\lim _{n} \varphi_{n}^{\prime}(z) /\left[\varphi_{n}(z)\right]^{r+1}=0$ l. $u$. in $|z|>1$. 
If we suppose that the assertion is true for $j$, then,

$$
\begin{aligned}
\left(\frac{\varphi_{n}^{(j)}(z)}{\left[\varphi_{n}(z)\right]^{r+1}}\right)^{\prime} & =\frac{\varphi_{n}^{(j+1)}(z)}{\left[\varphi_{n}(z)\right]^{r+1}}-(r+1) \frac{\varphi_{n}^{(j)}(z) \varphi_{n}^{\prime}(z)}{\left[\varphi_{n}(z)\right]^{r+2}} \\
& =\frac{\varphi_{n}^{(j+1)}(z)}{\left[\varphi_{n}(z)\right]^{r+1}}-(r+1) \frac{\varphi_{n}^{(j)}(z)}{\left[\varphi_{n}(z)\right]^{(r / 2)+1}} \cdot \frac{\varphi_{n}^{\prime}(z)}{\left[\varphi_{n}(z)\right]^{(r / 2)+1}} .
\end{aligned}
$$

From here, the statement holds using the induction hypothesis.

Proposition 3.5. Assume that $\mu \in \mathscr{N}$ and let $\mu_{1}$ be as in Lemma 3.3. Then

$$
\lim _{n} \frac{\varphi_{n}\left(z ; \mathrm{d} \mu_{1}\right)}{\varphi_{n+1}(z)}=\frac{\bar{c}}{|c|} \frac{1}{\bar{c} z-1}
$$

holds l. u. in $|z|>1$ if $|c| \geqslant 1$, and uniformly in $|z| \geqslant 1$ if $|c|>1$. Furthermore, we get

$$
\lim _{n} \frac{\kappa_{n}\left(\mathrm{~d} \mu_{1}\right)}{\kappa_{n+1}}=\frac{1}{|c|} .
$$

Proof. From (3.4), we deduce

$$
(z-c) \varphi_{n}\left(z ; \mathrm{d} \mu_{1}\right)=\left(1+\frac{\left|\varphi_{n+1}(c)\right|^{2}}{K_{n}(c, c)}\right)^{-1 / 2}\left[\varphi_{n+1}(z)-\frac{\varphi_{n+1}(c)}{K_{n}(c, c)} K_{n}(z, c)\right] .
$$

Notice that

$$
\lim _{n} \frac{\kappa_{n}\left(\mathrm{~d} \mu_{1}\right)}{\kappa_{n+1}}=\lim _{n}\left(1+\frac{\left|\varphi_{n+1}(c)\right|^{2}}{K_{n}(c, c)}\right)^{-1 / 2}=\frac{1}{|c|},
$$

from Lemma 2.6.

If $|z| \geqslant 1$, then

$$
\frac{(z-c) \varphi_{n}\left(z ; \mathrm{d} \mu_{1}\right)}{\varphi_{n+1}(z)}=\frac{\kappa_{n}\left(\mathrm{~d} \mu_{1}\right)}{\kappa_{n+1}}\left[1-\frac{\left|\varphi_{n+1}(c)\right|^{2}}{K_{n}(c, c)} \frac{K_{n}(z, c)}{\varphi_{n+1}(z) \overline{\varphi_{n+1}(c)}}\right] .
$$

Thus, by using Lemma 2.6 (ii),

$$
\lim _{n} \frac{(z-c) \varphi_{n}\left(z ; \mathrm{d} \mu_{1}\right)}{\varphi_{n+1}(z)}=\frac{1}{|c|}\left(1-\frac{|c|^{2}-1}{\bar{c} z-1}\right)=\frac{\bar{c}}{|c|} \frac{z-c}{\bar{c} z-1}
$$

l. $u$. in $|z|>1$ if $|c| \geqslant 1$, and uniformly in $|z| \geqslant 1$ if $|c|>1$. 
Proposition 3.6. Let $\mu \in \mathscr{N}, p \in \mathbb{N}$, and let $\mu_{p+1}$ be the measure given by $\mathrm{d} \mu_{p+1}=|z-c|^{2(p+1)} \mathrm{d} \mu$. Then, for the polynomials $\varphi_{n}\left(z ; \mathrm{d} \mu_{p+1}\right)$,

$$
\lim _{n} \frac{\varphi_{n}\left(z ; \mathrm{d} \mu_{p+1}\right)}{\varphi_{n+p+1}(z)}=\left(\frac{\bar{c}}{|c|} \frac{1}{\bar{c} z-1}\right)^{p+1}
$$

holds l. u. in $|z|>1$ if $|c| \geqslant 1$, and uniformly in $|z| \geqslant 1$ if $|c|>1$. Furthermore,

$$
\lim _{n} \frac{\kappa_{n}\left(\mathrm{~d} \mu_{p+1}\right)}{\kappa_{n+p+1}}=\frac{1}{|c|^{p+1}}
$$

Proof. Lemma 3.3 implies that $\mu_{j}$, given by $\mathrm{d} \mu_{j}=|z-c|^{2 j} \mathrm{~d} \mu$, belongs to the $\mathscr{N}$ class, for $j=0,1, \ldots, p+1$. From (3.5) and Proposition 3.5, we get

$$
\lim _{n} \frac{\kappa_{n}\left(\mathrm{~d} \mu_{p+1}\right)}{\kappa_{n+p+1}}=\lim _{n} \prod_{j}^{p} \frac{\kappa_{n+j}\left(\mathrm{~d} \mu_{p+1-j}\right)}{\kappa_{n+j+1}\left(\mathrm{~d} \mu_{p-j}\right)}=\frac{1}{|c|^{p+1}}
$$

and

$$
\lim _{n} \frac{\varphi_{n}\left(z ; \mathrm{d} \mu_{p+1}\right)}{\varphi_{n+p+1}(z)}=\prod_{j}^{p} \lim _{n} \frac{\varphi_{n+j}\left(z ; \mathrm{d} \mu_{p+1-j}\right)}{\varphi_{n+j+1}\left(z ; \mathrm{d} \mu_{p-j}\right)}=\left[\frac{c}{|c|} \frac{1}{(\bar{c} z-1)}\right]^{p+1} .
$$

Corollary 3.7. Assume that $\mu \in \mathscr{N},|c|>1$, and $p \in \mathbb{N}$. Then,

$$
\lim _{n}\left(1+\boldsymbol{\varphi}_{n+1}(c)[M(n)]^{-1}\left[\boldsymbol{\varphi}_{n+1}(c)\right]^{H}\right)=\lim _{n} \frac{\operatorname{det}[M(n+1)]}{\operatorname{det}[M(n)]}=|c|^{2(p+1)}
$$

holds, with $\boldsymbol{\varphi}_{n+1}(c)=\left(\varphi_{n+1}(c), \varphi_{n+1}^{\prime}(c), \ldots, \varphi_{n+1}^{(p)}(c)\right)$.

Proof. It is straightforward from (3.2) and Lemma 3.1.

Now, let $\mu$ be a probability measure with $\mathbb{T}$ as support. Let us assume that $\mu \in \mathscr{N}, c \in \mathbb{C}$, and $p \in \mathbb{N}$. Again, we write $\varphi_{n}\left(z ; \mathrm{d} \mu_{j}\right)$ the $n$th orthonormal polynomial with respect to $\mu_{j}$, and $K_{n}\left(z, y ; \mathrm{d} \mu_{j}\right)$ the corresponding $n$th kernel, $j=0,1, \ldots, p+1$. For fixed $m \in \mathbb{N}$, with $m \geqslant p$, we define the linear operator $\mathscr{F}_{m}: \mathbb{P}_{m+1} \rightarrow \mathbb{C}^{p+1}$ as

$$
\begin{aligned}
\mathscr{F}_{m}(P) & :=\int_{|z| 1} P(z) \overline{\mathbf{K}_{m+1}(z, c)} \mathrm{d} \mu(z)=\left(P(z), \mathbf{K}_{m+1}(z, c)\right)=\mathbf{P}(c) \\
& =\left(P(c), P^{\prime}(c), \ldots, P^{(p)}(c)\right) .
\end{aligned}
$$

It is easy to prove that

$$
\mathscr{F}_{m}[(z-c) P(z)]=\mathbf{P}(c) B
$$


for $P \in \mathbb{P}_{m}$, where $B$ is the matrix

$$
B:=\left(\begin{array}{ccccc}
0 & 1 & 0 & \ldots & 0 \\
0 & 0 & 2 & \ldots & 0 \\
\vdots & \vdots & \vdots & \vdots & \vdots \\
0 & 0 & 0 & \ldots & p \\
0 & 0 & 0 & \ldots & 0
\end{array}\right) \in \mathbb{R}^{(p+1, p+1)} .
$$

For each $m \geqslant p$ and each $j=0, \ldots, p$, we shall denote

$$
\begin{aligned}
& \mathbf{v}_{m+1}=\mathscr{F}_{m}\left[\varphi_{m+1}(z ; \mathrm{d} \mu)\right]=\boldsymbol{\varphi}_{m+1}(c ; \mathrm{d} \mu), \\
& \mathbf{v}_{m-j}=\mathscr{F}_{m}\left[\varphi_{m-j}\left(z ; \mathrm{d} \mu_{j+1}\right)\right]=\boldsymbol{\varphi}_{m-j}\left(c ; \mathrm{d} \mu_{j+1}\right), \\
& \mathbf{L}_{m-j}^{(k)}=\mathscr{F}_{m}\left[K_{m-j}^{(0, k)}\left(z, c ; \mathrm{d} \mu_{j}\right)\right]=\left(K_{m-j}^{(0, k)}\left(c, c ; \mathrm{d} \mu_{j}\right), \ldots, K_{m-j}^{(p, k)}\left(c, c ; \mathrm{d} \mu_{j}\right)\right), \\
& \mathbf{L}_{m-j}=\mathbf{L}_{m-j}^{(0)} .
\end{aligned}
$$

Notice that $\mathbf{L}_{m}^{(k)}$ is the $k$ th row $(0 \leqslant k \leqslant p)$ of the matrix $M(m)$.

From (3.4), for $j=0,1, \ldots, p$, we can put

$$
(z-c) \varphi_{m-j}\left(z ; \mathrm{d} \mu_{j+1}\right)=\alpha_{m-j}^{(j)}\left[\varphi_{m-j+1}\left(z ; \mathrm{d} \mu_{j}\right)-\beta_{m-j}^{(j)} K_{m-j}\left(z, c ; \mathrm{d} \mu_{j}\right)\right],
$$

with

$$
\alpha_{m-j}^{(j)}=\left(1+\frac{\left|\varphi_{m-j+1}\left(c ; \mathrm{d} \mu_{j}\right)\right|^{2}}{K_{m-j}\left(c, c ; \mathrm{d} \mu_{j}\right)}\right)^{-1 / 2}, \quad \beta_{m-j}^{(j)}=\frac{\varphi_{m-j+1}\left(c ; \mathrm{d} \mu_{j}\right)}{K_{m-j}\left(c, c ; \mathrm{d} \mu_{j}\right)} .
$$

Thus, by applying $\mathscr{\mathscr { F }}_{m}$

$$
\mathbf{v}_{m-j} B=\alpha_{m-j}^{(j)}\left(\mathbf{v}_{m-j+1}-\beta_{m-j}^{(j)} \mathbf{L}_{m-j}\right) .
$$

By iteration, we get

$$
\mathbf{v}_{m+1}=\frac{\mathbf{v}_{m-j} B^{j+1}}{\alpha_{m-j}^{(j)}, \ldots, \alpha_{m}^{(0)}}+\frac{\beta_{m-j}^{(j)}}{\alpha_{m-j+1}^{(j-1)}, \ldots, \alpha_{m}^{(0)}} \mathbf{L}_{m-j} B^{j}+\cdots+\beta_{m}^{(0)} \mathbf{L}_{m} .
$$

Since $B^{p+1}=0$, (3.6) becomes

$$
\mathbf{v}_{m+1}=\frac{\beta_{m-p}^{(p)}}{\alpha_{m-p+1}^{(p-1)}, \ldots, \alpha_{m}^{(0)}} \mathbf{L}_{m-p} B^{p}+\cdots+\frac{\beta_{m-1}^{(1)}}{\alpha_{m}^{(0)}} \mathbf{L}_{m-1} B+\beta_{m}^{(0)} \mathbf{L}_{m}
$$

for $j=p$.

On the other hand, for the kernel polynomials we have (see [5])

$$
\begin{aligned}
(z-c) \overline{(y-c)} K_{m-j-1}\left(z, y ; \mathrm{d} \mu_{j+1}\right)= & K_{m-j}\left(z, y ; \mathrm{d} \mu_{j}\right) \\
& -\frac{K_{m-j}\left(z, c ; \mathrm{d} \mu_{j}\right) K_{m-j}\left(c, y ; \mathrm{d} \mu_{j}\right)}{K_{m-j}\left(c, c ; \mathrm{d} \mu_{j}\right)},
\end{aligned}
$$


from where, computing the $k$ th derivative in $z=c$ and taking conjugate, we obtain

$$
\begin{aligned}
k(y-c) K_{m-j-1}^{(0, k-1)}\left(y, c ; \mathrm{d} \mu_{j+1}\right)= & K_{m-j}^{(0, k)}\left(y, c ; \mathrm{d} \mu_{j}\right) \\
& -\frac{K_{m-j}\left(y, c ; \mathrm{d} \mu_{j}\right) K_{m-j}^{(0, k)}\left(c, c ; \mathrm{d} \mu_{j}\right)}{K_{m-j}\left(c, c ; \mathrm{d} \mu_{j}\right)} .
\end{aligned}
$$

Then, applying $\mathscr{F}_{m}$, we get

$$
k \mathbf{L}_{m-j-1}^{(k-1)} B=\mathbf{L}_{m-j}^{(k)}-\delta_{m-j}^{(k)} \mathbf{L}_{m-j},
$$

with

$$
\delta_{m-j}^{(k)}=\frac{K_{m-j}^{(0, k)}\left(c, c ; \mathrm{d} \mu_{j}\right)}{K_{m-j}\left(c, c ; \mathrm{d} \mu_{j}\right)} .
$$

Proposition 3.8. If $j=0,1, \ldots, p$, then the following statements hold

(i) $\mathbf{L}_{m-j} B^{j}[M(m)]^{-1}\left[\mathbf{x} B^{i}\right]^{H}=0$, for all $i \geqslant j+1$ and for all $\mathbf{x} \in \mathbb{C}^{p+1}$.

(ii) $\mathbf{L}_{m-j} B^{j}[M(m)]^{-1}\left[\mathbf{L}_{m-j} B^{j}\right]^{H}=K_{m-j}\left(c, c ; \mathrm{d} \mu_{j}\right)$.

Proof. (i) For $j=0$, we have

$$
\mathbf{L}_{m}=\left(K_{m}(c, c ; \mathrm{d} \mu), \ldots, K_{m}^{(p, 0)}(c, c ; \mathrm{d} \mu)\right),
$$

and

$$
\mathbf{L}_{m}[M(m)]^{-1}=(1,0, \ldots, 0) .
$$

Thus, the statement follows immediately. Assume it is true for $0,1, \ldots, j-1$. Then, if $k=1$, we obtain from (3.8)

$$
\begin{aligned}
\mathbf{L}_{m-j} B^{j}[M(m)]^{-1}\left[\mathbf{x} B^{i}\right]^{H}= & \mathbf{L}_{m-j+1}^{(1)} B^{j-1}[M(m)]^{-1}\left[\mathbf{x} B^{i}\right]^{H} \\
& -\delta_{m-j+1}^{(1)} \mathbf{L}_{m-j+1} B^{j-1}[M(m)]^{-1}\left[\mathbf{x} B^{i}\right]^{H} .
\end{aligned}
$$

The induction hypothesis gives

$$
\mathbf{L}_{m-j} B^{j}[M(m)]^{-1}\left[\mathbf{x} B^{i}\right]^{H}=\mathbf{L}_{m-j+1}^{(1)} B^{j-1}[M(m)]^{-1}\left[\mathbf{x} B^{i}\right]^{H},
$$

and, for $k=2, \ldots, j$ in $(3.8)$,

$$
\begin{aligned}
\mathbf{L}_{m-j} B^{j}[M(m)]^{-1}\left[\mathbf{x} B^{i}\right]^{H} & =\frac{1}{2} \mathbf{L}_{m-j+2}^{(2)} B^{j-2}[M(m)]^{-1}\left[\mathbf{x} B^{i}\right]^{H}=\cdots \\
& =\frac{1}{j !} \mathbf{L}_{m}^{(j)}[M(m)]^{-1}\left[\mathbf{x} B^{i}\right]^{H} \\
& =\frac{1}{j !} \overbrace{0, \ldots, 0}^{j-1}, 1,0, \ldots, 0)\left(B^{\mathrm{T}}\right)^{i} \mathbf{x}^{H}=0
\end{aligned}
$$


follows, taking into account that

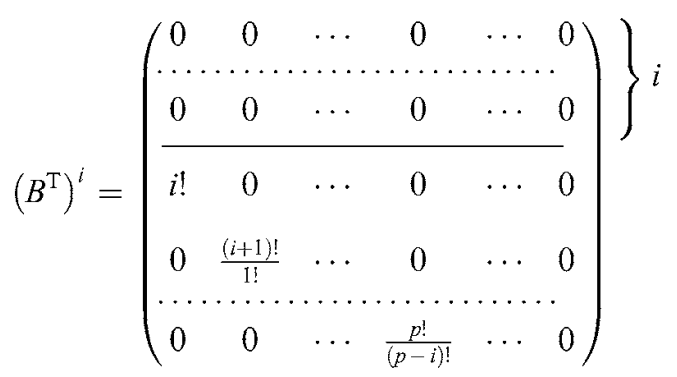

(ii) For $j=0$, we have

$$
\mathbf{L}_{m}[M(m)]^{-1} \mathbf{L}_{m}^{H}=(1,0, \ldots, 0) \mathbf{L}_{m}^{H}=K_{m}(c, c ; \mathrm{d} \mu) .
$$

Assume that our hypothesis is true for $0,1, \ldots, j-1$. From (3.8) and the first statement,

$$
\begin{aligned}
\mathbf{L}_{m-j} B^{j}[M(m)]^{-1}\left[\mathbf{L}_{m-j} B^{j}\right]^{H} & =\mathbf{L}_{m-j+1}^{(1)} B^{j-1}[M(m)]^{-1}\left[\mathbf{L}_{m-j} B^{j}\right]^{H}=\ldots \\
& =\frac{1}{j !} \mathbf{L}_{m}^{(j)}[M(m)]^{-1}\left[\mathbf{L}_{m-j} B^{j}\right]^{H} \\
& =\frac{1}{j !}(\overbrace{0, \ldots, 0}^{j-1}, 1,0, \ldots, 0)\left(B^{\mathrm{T}}\right)^{j} \mathbf{L}_{m-j}^{H} \\
& =K_{m-j}\left(c, c ; \mathrm{d} \mu_{j}\right)
\end{aligned}
$$

follows, for $k=1, \ldots, j$.

Corollary 3.9. $\left\{\mathbf{L}_{m-j} B^{j}\right\}_{j}^{p}{ }_{0}$ is an orthogonal basis in $\mathbb{C}^{p+1}$ for the inner product $\mathbf{x}[M(m)]^{-1} \mathbf{y}^{H}$.

Notice that (3.7) gives also an orthogonal decomposition for $\mathbf{v}_{m+1}$ (with respect to the inner product $\left.\mathbf{x}[M(m)]^{-1} \mathbf{y}^{H}\right)$ for $j=0, \ldots, p-1$.

Corollary 3.10. If $\mu \in \mathscr{N}$ and $|c| \geqslant 1$, then

$$
\lim _{m}\left[\mathbf{v}_{m-i+1} B^{i}[M(m)]^{-1}\left[\mathbf{v}_{m-i+1} B^{i}\right]^{H}\right]=|c|^{2(p-i+1)}-1
$$

holds for $i=0, \ldots, p$. 
Proof. Because the orthogonality of the decomposition (3.6), we have

$$
\begin{aligned}
\mathbf{v}_{m+1}[M(m)]^{-1} \mathbf{v}_{m+1}^{H}= & \frac{\mathbf{v}_{m-j} B^{j+1}[M(m)]^{-1}\left[\mathbf{v}_{m-j} B^{j+1}\right]^{H}}{\left|\alpha_{m-j}^{(j)}, \ldots, \alpha_{m}^{(0)}\right|^{2}} \\
& +\frac{\left|\beta_{m-j}^{(j)}\right|^{2} \mathbf{L}_{m-j} B^{j}[M(m)]^{-1}\left[\mathbf{L}_{m-j} B^{j}\right]^{H}}{\left|\alpha_{m-j+1}^{(j-1)}, \ldots, \alpha_{m}^{(0)}\right|^{2}}+\cdots \\
& +\left|\beta_{m}^{(0)}\right|^{2} \mathbf{L}_{m}[M(m)]^{-1} \mathbf{L}_{m}^{H} .
\end{aligned}
$$

But, Corollary 3.7 implies that

$$
\lim _{m}\left(\mathbf{v}_{m+1}[M(m)]^{-1} \mathbf{v}_{m+1}^{H}\right)=|c|^{2 p+2}-1 .
$$

On the other hand, $\lim _{m} \alpha_{m-k}^{(k)}=|c|^{-1}$ and, by Proposition 3.8 (ii),

$$
\left|\beta_{m-k}^{(k)}\right|^{2} \mathbf{L}_{m-k} B^{k}[M(m)]^{-1}\left[\mathbf{L}_{m-k} B^{k}\right]^{H}=\frac{\left|\varphi_{m-k+1}\left(c ; \mathrm{d} \mu_{k}\right)\right|^{2}}{K_{m-k}\left(c, c ; \mathrm{d} \mu_{k}\right)},
$$

$(k=0, \ldots j)$, which tends to $|c|^{2}-1$ when $m \rightarrow \infty$ (Lemma 2.6). Thus, we have

$$
\begin{aligned}
|c|^{2 p+2}-1= & |c|^{2 j+2} \lim _{m}\left[\mathbf{v}_{m-j} B^{j+1}[M(m)]^{-1}\left[\mathbf{v}_{m-j} B^{j+1}\right]^{H}\right] \\
& +|c|^{2 j}\left(|c|^{2}-1\right)+\cdots+|c|^{2}-1,
\end{aligned}
$$

and

$$
\lim _{m}\left[\mathbf{v}_{m-j} B^{j+1}[M(m)]^{-1}\left[\mathbf{v}_{m-j} B^{j+1}\right]^{H}\right]=|c|^{2(p-j)}-1
$$

holds for $j=0, \ldots, p-1$. So, the Proposition is true for $i=1, \ldots, p$. For $i=0$, we recover Corollary 3.7.

Lemma 3.11. Let $\mathbf{x}_{1}, \ldots, \mathbf{x}_{k}$ be vectors in a complex vector space with an inner product $[\cdot, \cdot]$. Then,

$$
\sum_{i, j}^{k}\left|\left[\mathbf{x}_{i}, \mathbf{x}_{j}\right]\right| \leqslant k \sum_{i=1}^{k}\left[\mathbf{x}_{i}, \mathbf{x}_{i}\right] .
$$

Proof. By the Cauchy Schwarz's inequality we have

$$
\left|\left[\mathbf{x}_{i}, \mathbf{x}_{j}\right]\right| \leqslant\left(\left[\mathbf{x}_{i}, \mathbf{x}_{i}\right] \cdot\left[\mathbf{x}_{j}, \mathbf{x}_{j}\right]\right)^{1 / 2} \leqslant \frac{1}{2}\left(\left[\mathbf{x}_{i}, \mathbf{x}_{i}\right]+\left[\mathbf{x}_{j}, \mathbf{x}_{j}\right]\right) .
$$

Hence,

$$
\sum_{i, j}^{k}\left|\left[\mathbf{x}_{i}, \mathbf{x}_{j}\right]\right| \leqslant \frac{1}{2} \sum_{i, j 1}^{k}\left(\left[\mathbf{x}_{i}, \mathbf{x}_{i}\right]+\left[\mathbf{x}_{j}, \mathbf{x}_{j}\right]\right)=k \sum_{i=1}^{k}\left[\mathbf{x}_{i}, \mathbf{x}_{i}\right] .
$$


Lemma 3.12. If $\mu \in \mathscr{N}$ and $|c|>1$, then for fixed numbers $i \in \mathbb{Z}$ and $j, k, h \in \mathbb{N}$, the following statements are fulfilled

(i) $\left|\varphi_{n+i}^{(k)}\left(c ; \mathrm{d} \mu_{j}\right)\right|=\mathcal{O}\left(\left|\varphi_{n}^{(k)}(c)\right|\right)$,

(ii) $\left|K_{n+i}^{(k, h)}\left(c, c ; \mathrm{d} \mu_{j}\right)\right|=\mathcal{O}\left(\left|\varphi_{n}^{(k)}(c) \varphi_{n}^{(h)}(c)\right|\right)$.

Proof. (i) If $z=c(|c|>1)$ in Proposition 3.6, then

$$
\lim _{n} \frac{\varphi_{n}\left(c ; \mathrm{d} \mu_{j+1}\right)}{\varphi_{n+j+1}(c)}=\left(\frac{\bar{c}}{|c|} \cdot \frac{1}{|c|^{2}-1}\right)^{j+1} .
$$

On the other hand, from Lemma 2.4 we have

$$
\frac{\varphi_{n}^{(k)}\left(c ; \mathrm{d} \mu_{j+1}\right)}{\varphi_{n+j+1}^{(k)}(c)}=\left.\frac{\varphi_{n+j+1}^{(k-1)}(c)}{\varphi_{n+j+1}^{(k)}(c)}\left(\frac{\varphi_{n}^{(k-1)}\left(z ; \mathrm{d} \mu_{j+1}\right)}{\varphi_{n+j+1}^{(k-1)}(z)}\right)^{\prime}\right|_{z c}+\frac{\varphi_{n}^{(k-1)}\left(c ; \mathrm{d} \mu_{j+1}\right)}{\varphi_{n+j+1}^{(k-1)}(c)} .
$$

Since Lemma 2.5 (i) and the uniform convergence of $\left(\varphi_{n}^{(k-1)}\left(z ; \mathrm{d} \mu_{j+1}\right)\right) / \varphi_{n+j+1}^{(k-1)}(z)$, we can write

$$
\lim _{n} \frac{\varphi_{n}^{(k)}\left(c ; \mathrm{d} \mu_{j+1}\right)}{\varphi_{n+j+1}^{(k)}(c)}=\lim _{n} \frac{\varphi_{n}^{(k-1)}\left(c ; \mathrm{d} \mu_{j+1}\right)}{\varphi_{n+j+1}^{(k-1)}(c)}=\left(\frac{\bar{c}}{|c|} \cdot \frac{1}{|c|^{2}-1}\right)^{j+1} .
$$

Furthermore, $\lim _{n}\left(\varphi_{n+i}^{(k)}(z)\right) / \varphi_{n}^{(k)}(z)=z^{i} l . u$. in $|z|>1$. Then, the first statement follows.

(ii) Choose $z=c$ in Lemma 2.6 (ii). Then,

$$
\lim _{n} \frac{K_{n+i}^{(k, h)}\left(c, c ; \mathrm{d} \mu_{j}\right)}{\varphi_{n+i+1}^{(k)}\left(c ; \mathrm{d} \mu_{j}\right) \overline{\varphi_{n+i+1}^{(h)}\left(c ; \mathrm{d} \mu_{j}\right)}}=\frac{1}{|c|^{2}-1},
$$

and it is enough to use (i).

Theorem 3.13. If $\mu \in \mathscr{N}$ and $|c|>1$, the spectral radius of $[M(n)]^{-1}$, $\rho\left([M(n)]^{-1}\right)$, tends to zero when $n \rightarrow \infty$.

Proof. For each $n \in \mathbb{N}$, denote $\mathbf{V}_{j}^{(n)}=\mathbf{L}_{n+j} B^{p-j}(j=0, \ldots, p)$. Thus (Corollary 3.9), $\left(\mathbf{V}_{j}^{(n)}\right)_{j \quad 0_{0}}^{p}$ is an orthogonal basis in $\mathbb{C}^{p+1}$ with respect to the inner product $\mathbf{x}[M(n+p)]^{-1} \mathbf{y}^{H}$.

It is necessary to prove that $\lim _{n}\left(\mathbf{x}[M(n)]^{-1} \mathbf{x}^{H}\right) /\|\mathbf{x}\|^{2}=0$, for all $\mathbf{x} \neq 0$. However, if $\left(\mathbf{u}_{j}^{(n)}\right)_{j}^{p}$ is an orthonormal basis of $\mathbb{C}^{p+1}$ for each $n$, and $\mathbf{x}=\sum_{j \quad 0}^{p} x_{j n} \mathbf{u}_{j}^{(n)}$, from Lemma 3.11 we have 


$$
\begin{aligned}
\frac{\mathbf{x}[M(n)]^{-1} \mathbf{x}^{H}}{\|\mathbf{x}\|^{2}} & =\frac{\sum_{i, j} x_{i n} \mathbf{u}_{i}^{(n)}[M(n)]^{-1}\left[x_{j n} \mathbf{u}_{j}^{(n)}\right]^{H}}{\sum_{i}\left|x_{i n}\right|^{2}} \\
& \leqslant(p+1) \frac{\sum_{i}\left|x_{i n}\right|^{2} \mathbf{u}_{i}^{(n)}[M(n)]^{-1}\left[\mathbf{u}_{i}^{(n)}\right]^{H}}{\sum_{i}\left|x_{i n}\right|^{2}} \\
& \leqslant(p+1) \sum_{i} \mathbf{u}_{i}^{(n)}[M(n)]^{-1}\left[\mathbf{u}_{i}^{(n)}\right]^{H} .
\end{aligned}
$$

Hence, it is enough to prove that $\lim _{n} \mathbf{u}_{i}^{(n)}[M(n)]^{-1}\left[\mathbf{u}_{i}^{(n)}\right]^{H}=0$, or

$$
\lim _{n} \frac{\mathbf{u}_{i}^{(n)}[M(n)]^{-1}\left[\mathbf{u}_{i}^{(n)}\right]^{H}}{\left\|\mathbf{u}_{i}^{(n)}\right\|^{2}}=0
$$

when $\left(\mathbf{u}_{i}^{(n)}\right)_{i \quad 0}^{p}$ is orthogonal (for each $n$ ).

So, we will orthogonalize $\left(\mathbf{V}_{j}^{(n)}\right)$ by using the Gram Schmidt method, and, at once, we will study (3.9). Thus, let $\left(\mathbf{u}_{j}^{(n)}\right)$ be the orthogonal basis such that

$$
\mathbf{u}_{0}^{(n)}=\mathbf{V}_{0}^{(n)}, \mathbf{u}_{j}^{(n)}=\mathbf{V}_{j}^{(n)}-\sum_{k 0}^{j-1} \theta_{k}^{(j)} \mathbf{u}_{k}^{(n)} \quad(j=1, \ldots, p),
$$

where the $\theta_{k}^{(j)}$, s (which depend on $n$ ) are given by

$$
\theta_{k}^{(j)}=\frac{\mathbf{V}_{j}^{(n)}\left[\mathbf{u}_{k}^{(n)}\right]^{H}}{\left\|\mathbf{u}_{k}^{(n)}\right\|^{2}}, \quad k=0, \ldots, j-1 ; j=1, \ldots, p .
$$

If we consider

$$
\begin{aligned}
& \mathbf{V}_{j}^{(n)} \\
& =[\overbrace{0, \ldots, 0}^{p-j},(p-j) ! K_{n+j}\left(c, c ; \mathrm{d} \mu_{p-j}\right), \ldots, \frac{(p-k) !}{(j-k) !} K_{n+j}^{(j-k, 0)}\left(c, c ; \mathrm{d} \mu_{p-j}\right), \ldots],
\end{aligned}
$$

then

$$
\mathbf{u}_{j}^{(n)}=[\overbrace{0, \ldots, 0}^{p-j},(p-j) ! K_{n+j}\left(c, c ; \mathrm{d} \mu_{p-j}\right), 0, \ldots, 0]
$$

follows for $j=0, \ldots, p$. From here, we have

$$
\theta_{k}^{(j)}=\frac{1}{(j-k) !} \frac{K_{n+j}^{(j-k, 0)}\left(c, c ; \mathrm{d} \mu_{p-j}\right)}{K_{n+k}\left(c, c ; \mathrm{d} \mu_{p-k}\right)}, \quad k=0, \ldots, j-1 ; j=1, \ldots, p .
$$


By using Lemma 3.12, we obtain

$$
\left|\theta_{k}^{(j)}\right|=\mathcal{O}\left(\left|\frac{\varphi_{n}^{(j-k)}(c)}{\varphi_{n}(c)}\right|\right)
$$

and $\left\|\mathbf{u}_{j}^{(n)}\right\|=\mathcal{O}\left(\left|\varphi_{n}(c)\right|^{2}\right)$. Also, $\lim _{n}\left(\varphi_{n}^{(j-k)}(c)\right) / \varphi_{n}^{(j)}(c)=0(1 \leqslant k \leqslant j)$, from where $\left|\theta_{k}^{(j)}\right| \leqslant\left|\theta_{0}^{(j)}\right|$ for $k=0, \ldots, j$ and $n$ large enough.

We will use induction to prove that

$$
\frac{\mathbf{u}_{j}^{(n)}[M(n+p)]^{-1}\left[\mathbf{u}_{j}^{(n)}\right]^{H}}{\left\|\mathbf{u}_{j}^{(n)}\right\| \|^{2}}=\mathcal{O}\left(\frac{\prod_{h}^{j} 0\left|\varphi_{n}^{(j-h)}(c)\right|^{2}}{\left|\varphi_{n}(c)\right|^{2(j+2)}}\right)
$$

with $j=0, \ldots, p$, or, equivalently, taking into account that $\left\|\mathbf{u}_{j}^{(n)}\right\|=$ $\mathcal{O}\left(\left|\varphi_{n}(c)\right|^{2}\right)$,

$$
\mathbf{u}_{j}^{(n)}[M(n+p)]^{-1}\left[\mathbf{u}_{j}^{(n)}\right]^{H}=\mathcal{O}\left(\frac{\prod_{h}^{j} 0\left|\varphi_{n}^{(j-h)}(c)\right|^{2}}{\left|\varphi_{n}(c)\right|^{2 j}}\right) .
$$

In fact, if $j=0$,

$$
\begin{aligned}
\mathbf{u}_{0}^{(n)}[M(n+p)]^{-1}\left[\mathbf{u}_{0}^{(n)}\right]^{H} & =\mathbf{V}_{0}^{(n)}[M(n+p)]^{-1}\left[\mathbf{V}_{0}^{n}\right]^{H}=K_{n+j}\left(c, c ; \mathrm{d} \mu_{p}\right) \\
& =\mathcal{O}\left(\left|\varphi_{n}(c)\right|^{2}\right),
\end{aligned}
$$

according to Corollary 3.10. Assume that the hypothesis is true for $j-1$. Then,

$$
\begin{aligned}
\mathbf{u}_{j}^{(n)}[M(n+p)]^{-1}\left[\mathbf{u}_{j}^{(n)}\right]^{H}= & \mathbf{V}_{j}^{(n)}[M(n+p)]^{-1}\left[\mathbf{V}_{j}^{(n)}\right]^{H} \\
& +\sum_{k, i}^{j-1} \theta_{k}^{(j)} \mathbf{u}_{k}^{(n)}[M(n+p)]^{-1}\left[\theta_{i}^{(j)} \mathbf{u}_{i}^{(n)}\right]^{H} .
\end{aligned}
$$

Since Lemma (3.11) and (3.10),

$$
\begin{aligned}
\mathbf{u}_{j}^{(n)}[M(n+p)]^{-1}\left[\mathbf{u}_{j}^{(n)}\right]^{H} \leqslant & K_{n+j}\left(c, c ; \mathrm{d} \mu_{p-j}\right) \\
& +j\left|\theta_{0}^{(j)}\right|^{2} \sum_{k 0}^{j-1} \mathbf{u}_{k}^{(n)}[M(n+p)]^{-1}\left[\mathbf{u}_{k}^{(n)}\right]^{H} \\
= & \mathcal{O}\left(\left|\varphi_{n}(c)\right|^{2}\right)+\mathcal{O}\left(\left|\frac{\varphi_{n}^{(j)}(c)}{\varphi_{n}(c)}\right|^{2}\right) \\
& \times \sum_{k 0}^{j-1} \mathcal{O}\left(\frac{\prod_{h 0}^{k}\left|\varphi_{n}^{(k-h)}(c)\right|^{2}}{\left|\varphi_{n}(c)\right|^{2 k}}\right) .
\end{aligned}
$$


Keeping in mind that $\lim _{n}\left(\varphi_{n}^{(j-k)}(c)\right) / \varphi_{n}^{(j)}(c)=0$, for $1 \leqslant k \leqslant j$, the induction process is finished. Hence,

$$
\begin{aligned}
\rho\left([M(n+p)]^{-1}\right) & =\mathcal{O}\left(\frac{\prod_{h 0}^{p}\left|\varphi_{n}^{(p-h)}(c)\right|^{2}}{\left|\varphi_{n}(c)\right|^{2(p+2)}}\right) \\
& =\mathcal{O}\left(\prod_{h}^{p}\left|\frac{\varphi_{n}^{(p-h)}(c)}{\left[\varphi_{n}(c)\right]^{1+r}}\right|^{2}\right),
\end{aligned}
$$

with $r=1 /(p+1)>0$, and, thus, $\rho\left([M(n+p)]^{-1}\right)$ tends to zero (Lemma 3.4).

Now, we will explain not only how to expand the polynomial $\varphi_{n-p}\left(z ; \mathrm{d} \mu_{p+1}\right)$ in terms of $\varphi_{n+1}(z)$ and $\varphi_{n+1}^{*}(z)$, but also to find several asymptotic properties for the expansion coefficients.

Let us consider (3.3)

$$
\begin{aligned}
(z-c)^{p+1} \varphi_{n-p}\left(z ; \mathrm{d} \mu_{p+1}\right)= & \frac{\kappa_{n-p}\left(\mathrm{~d} \mu_{p+1}\right)}{\kappa_{n+1}}\left(\varphi_{n+1}(z)\right. \\
& \left.-\boldsymbol{\varphi}_{n+1}(c)[M(n)]^{-1}\left[\mathbf{K}_{n}(z, c)\right]^{\mathrm{T}}\right),
\end{aligned}
$$

where $\boldsymbol{\varphi}_{n+1}(c):=\boldsymbol{\varphi}_{n+1}(c ; \mathrm{d} \mu), \mathbf{K}_{n}(z, c):=\mathbf{K}_{n}(z, c ; \mathrm{d} \mu), \kappa_{n+1}:=\kappa_{n+1}(\mathrm{~d} \mu)$, and $c \in \mathbb{C}$. Define

$$
\mathbf{T}_{n}(z)=\left[\tau_{n 0}(z), \tau_{n 1}(z), \ldots, \tau_{n p}(z)\right]^{\mathrm{T}}:=[M(n)]^{-1}\left[\mathbf{K}_{n}(z, c)\right]^{\mathrm{T}} .
$$

Notice that $\left.\frac{\partial^{j}}{\partial z^{j}}\left[\mathbf{K}_{n}(z, c)\right]^{\mathrm{T}}\right|_{z c}$ is the $j$ th column of $M(n)(0 \leqslant j \leqslant p)$. It follows that

$$
\tau_{n i}^{(j)}(c)=\delta_{i j}, \quad i, j=0, \ldots, p,
$$

and, for $n=p$,

$$
\tau_{p i}(z)=\frac{(z-c)^{i}}{i !}, \quad i=0, \ldots, p .
$$

Substituting (3.13) in (3.12), we have

$$
(z-c)^{p+1} \varphi_{n-p}\left(z ; \mathrm{d} \mu_{p+1}\right)=\frac{\kappa_{n-p}\left(\mathrm{~d} \mu_{p+1}\right)}{\kappa_{n+1}}\left[\varphi_{n+1}(z)-\boldsymbol{\varphi}_{n+1}(c) \mathbf{T}_{n}(z)\right],
$$

for all $n \geqslant p$. Remark that, for $n=p$, the bracket in the second member is the difference between $\varphi_{p+1}(z)$ and his Taylor polynomial of degree $p$.

Proposition 3.14. The sequence $\left(\mathbf{T}_{n}(z)\right)_{n \geqslant p}$ satisfies the following recurrence relation 


$$
\begin{aligned}
\mathbf{T}_{n+1}(z)= & \mathbf{T}_{n}(z)+\frac{\kappa_{n+1}(\mathrm{~d} \mu)}{\kappa_{n-p}\left(\mathrm{~d} \mu_{p+1}\right)}(z-c)^{p+1} \varphi_{n-p}\left(z ; \mathrm{d} \mu_{p+1}\right) \\
& \times[M(n+1)]^{-1}\left[\boldsymbol{\varphi}_{n+1}(c)\right]^{H} .
\end{aligned}
$$

Proof. We have

$$
\begin{aligned}
M(n+1)\left[\mathbf{T}_{n+1}(z)-\mathbf{T}_{n}(z)\right]= & M(n+1) \mathbf{T}_{n+1}(z) \\
& -\left[M(n)+\left[\boldsymbol{\varphi}_{n+1}(c)\right]^{H} \boldsymbol{\varphi}_{n+1}(c)\right] \mathbf{T}_{n}(z) .
\end{aligned}
$$

Then, because (3.13), it follows

$$
\begin{aligned}
M(n+1)\left[\mathbf{T}_{n+1}(z)-\mathbf{T}_{n}(z)\right]= & {\left[\mathbf{K}_{n+1}(z, c)\right]^{\mathrm{T}}-\left[\mathbf{K}_{n}(z, c)\right]^{\mathrm{T}}-\left[\boldsymbol{\varphi}_{n+1}(c)\right]^{H} } \\
& \times \boldsymbol{\varphi}_{n+1}(c) \mathbf{T}_{n}(z) \\
= & {\left[\boldsymbol{\varphi}_{n+1}(c)\right]^{H}\left[\varphi_{n+1}(z)-\boldsymbol{\varphi}_{n+1}(c) \mathbf{T}_{n}(z)\right], }
\end{aligned}
$$

and, from (3.14), we can conclude the proof.

Applying the operator $\mathscr{F}_{n}$ in the Christoffel Darboux formula (2.1), we obtain

$$
\begin{aligned}
\mathscr{F}_{n}\left[(1-\bar{y} z) K_{n}(z, y)\right] & =\overline{\mathbf{K}_{n}(y, c)}[(1-\bar{y} c) I-\bar{y} B] \\
& =\overline{\varphi_{n+1}^{*}(y)} \boldsymbol{\varphi}_{n+1}^{*}(c)-\overline{\varphi_{n+1}(y)} \boldsymbol{\varphi}_{n+1}(c),
\end{aligned}
$$

where

$$
\boldsymbol{\varphi}_{n+1}^{*}(c):=\left[\varphi_{n+1}^{*}(c), \varphi_{n+1}^{*^{\prime}}(c), \ldots, \varphi_{n+1}^{*(p)}(c)\right] .
$$

So, if $z \bar{c} \neq 1$,

$$
\overline{\mathbf{K}_{n}(z, c)}=\frac{\overline{\varphi_{n+1}^{*}(z)} \boldsymbol{\varphi}_{n+1}^{*}(c)-\overline{\varphi_{n+1}(z)} \boldsymbol{\varphi}_{n+1}(c)}{1-c \bar{z}}\left[I-\frac{\bar{z}}{1-c \bar{z}} B\right]^{-1}
$$

is obtained. Remember that $B$ is nilpotent $\left(B^{p+1}=0\right)$. Thus,

$$
\mathbf{K}_{n}(z, c)=\frac{\varphi_{n+1}^{*}(z) \overline{\boldsymbol{\varphi}_{n+1}^{*}(c)}-\varphi_{n+1}(z) \overline{\boldsymbol{\varphi}_{n+1}(c)}}{1-z \bar{c}} \sum_{k=0}^{p}\left(\frac{z}{1-z \bar{c}} B\right)^{k} .
$$

Proposition 3.15. If $c \neq 0$, then, for each $n \geqslant p+1$ there are two polynomials $P(z ; n)$ and $Q(z ; n)$, such that

$$
(1-\bar{c} z)^{p+1}(z-c)^{p+1} \varphi_{n-p-1}\left(z ; \mathrm{d} \mu_{p+1}\right)=P(z ; n) \varphi_{n}(z)+Q(z ; n) \varphi_{n}^{*}(z) .
$$

with $\operatorname{deg} P(z ; n)=p+1$ and $\operatorname{deg} Q(z ; n) \leqslant p$. 
Proof. Carrying (3.15) on (3.12), we obtain

$$
\begin{aligned}
& (z-c)^{p+1} \varphi_{n-p-1}\left(z ; \mathrm{d} \mu_{p+1}\right) \\
& =\frac{\kappa_{n-p-1}\left(\mathrm{~d} \mu_{p+1}\right)}{\kappa_{n}}\left[\varphi_{n}(z)\left(1+\sum_{k 0}^{p} \frac{z^{k}}{(1-\bar{c} z)^{k+1}} \boldsymbol{\varphi}_{n}(c)[M(n-1)]^{-1}\left[\boldsymbol{\varphi}_{n}(c) B^{k}\right]^{H}\right)\right. \\
& \left.-\varphi_{n}^{*}(z) \sum_{k 0}^{p} \frac{z^{k}}{(1-\bar{c} z)^{k+1}} \boldsymbol{\varphi}_{n}(c)[M(n-1)]^{-1}\left[\boldsymbol{\varphi}_{n}^{*}(c) B^{k}\right]^{H}\right] .
\end{aligned}
$$

From here, we get

$$
\begin{gathered}
\begin{aligned}
P(z ; n)=\frac{\kappa_{n-p-1}\left(\mathrm{~d} \mu_{p+1}\right)}{\kappa_{n}}[ & (1-\bar{c} z)^{p+1}+\sum_{k}^{p} z^{k}(1-\bar{c} z)^{p-k} \boldsymbol{\varphi}_{n}(c)[M(n-1)]^{-1} \\
& \left.\times\left[\boldsymbol{\varphi}_{n}(c) B^{k}\right]^{H}\right]
\end{aligned} \\
Q(z ; n)=-\frac{\kappa_{n-p-1}\left(\mathrm{~d} \mu_{p+1}\right)}{\kappa_{n}} \sum_{k 0}^{p} z^{k}(1-\bar{c} z)^{p-k} \boldsymbol{\varphi}_{n}(c)[M(n-1)]^{-1}\left[\boldsymbol{\varphi}_{n}^{*}(c) B^{k}\right]^{H} .
\end{gathered}
$$

Theorem 3.16. Let $\mu \in \mathscr{N}$ and $|c|>1$. Then, for the polynomials $P(z ; n)$ and $Q(z ; n)$ given in Proposition 3.15,

$$
\lim _{n} P(z ; n)=\left[\frac{\bar{c}}{|c|}(c-z)\right]^{p+1}, \quad \lim _{n} Q(z ; n)=0
$$

l. u. in $\mathbb{C}$.

Proof. If $p=0$, then

$$
(1-\bar{c} z)(z-c) \varphi_{n-1}\left(z ; \mathrm{d} \mu_{1}\right)=\hat{P}(z ; n) \varphi_{n}(z)+\hat{Q}(z ; n) \varphi_{n}^{*}(z),
$$

where

$$
\begin{aligned}
& \hat{P}(z ; n)=\frac{\kappa_{n-1}\left(\mathrm{~d} \mu_{1}\right)}{\kappa_{n}}\left[(1-\bar{c} z)+\frac{\left|\varphi_{n}(c)\right|^{2}}{K_{n-1}(c, c)}\right], \\
& \hat{Q}(z ; n)=-\frac{\kappa_{n-1}\left(\mathrm{~d} \mu_{1}\right)}{\kappa_{n}} \frac{\varphi_{n}(c) \overline{\varphi_{n}^{*}(c)}}{K_{n-1}(c, c)},
\end{aligned}
$$

and $\operatorname{deg} \hat{P}(z ; n)=1, \operatorname{deg} \hat{Q}(z ; n) \leqslant 0$.

From (2.2), Lemma 2.6 (i), and Proposition 3.5, the above statement for $\hat{P}(z ; n)$ and $\hat{Q}(z ; n)$ can be deduced in a straightforward way.

Assume that our assertion is true for $p-1$. There are two polynomials $\tilde{P}(z ; n)$ and $\tilde{Q}(z ; n)$ such that

$$
(1-\bar{c} z)^{p}(z-c)^{p} \varphi_{n-p}\left(z ; \mathrm{d} \mu_{p}\right)=\tilde{P}(z ; n) \varphi_{n}(z)+\tilde{Q}(z, n) \varphi_{n}^{*}(z),
$$


with $\operatorname{deg} \tilde{P}(z ; n)=p$ and $\operatorname{deg} \tilde{Q}(z ; n) \leqslant p-1$, and

$$
\lim _{n} \tilde{P}(z ; n)=\left[\frac{\bar{c}}{|c|}(c-z)\right]^{p}, \quad \lim _{n} \tilde{Q}(z ; n)=0,
$$

l. u. in $\mathbb{C}$.

But, if $\mu \in \mathscr{N}$, then also $\mu_{p} \in \mathscr{N}$. Hence, there exist two polynomials $D(z ; n)$ and $E(z ; n)$, with $\operatorname{deg} D(z ; n)=1, \operatorname{deg} E(z ; n) \leqslant 0$, such that

$$
\begin{aligned}
(1-\bar{c} z)(z-c) \varphi_{n-p-1}\left(z ; \mathrm{d} \mu_{p+1}\right)= & D(z ; n) \varphi_{n-p}\left(z ; \mathrm{d} \mu_{p}\right) \\
& +E(z ; n) \varphi_{n-p}^{*}\left(z ; \mathrm{d} \mu_{p}\right) .
\end{aligned}
$$

Then, $\lim _{n} D(z ; n)=(\bar{c} /|c|)(c-z), \lim _{n} E(z ; n)=0$ l. u. in $\mathbb{C}$.

Write $\tilde{P}^{*}(z ; n)=z^{p} \overline{\tilde{P}}(1 / z ; n)$ and $\tilde{Q}^{*}(z ; n)=z^{p-1} \overline{\tilde{Q}}(1 / z ; n)$. From (3.16), we have

$$
(1-\bar{c} z)^{p}(z-c)^{p} \varphi_{n-p}^{*}\left(z ; \mathrm{d} \mu_{p}\right)=\tilde{P}^{*}(z ; n) \varphi_{n}^{*}(z)+z \tilde{Q}^{*}(z ; n) \varphi_{n}(z),
$$

and, from (3.17), we get

$$
(1-\bar{c} z)^{p+1}(z-c)^{p+1} \varphi_{n-p-1}\left(z ; \mathrm{d} \mu_{p+1}\right)=P(z ; n) \varphi_{n}(z)+Q(z ; n) \varphi_{n}^{*}(z),
$$

with

$$
\begin{aligned}
& P(z ; n)=D(z ; n) \tilde{P}(z, n)+z E(z ; n) \tilde{Q}^{*}(z ; n), \\
& Q(z ; n)=D(z ; n) \tilde{Q}(z ; n)+E(z ; n) \tilde{P}^{*}(z ; n),
\end{aligned}
$$

and our results follow.

\section{Orthogonal polynomials related to a Sobolev-type inner product}

Let $\left(\varphi_{n}\right)_{n \in \mathbb{N}}$ be an orthonormal polynomial sequence related to a probability measure $\mu$ supported in $\mathbb{T}$ which induces an inner product $(\cdot, \cdot)$ on $\mathbb{P}$. For $c \in \mathbb{C}$ and $A \in \mathbb{C}^{(p+1, p+1)}(p \in \mathbb{N})$, we define in $\mathbb{P}$ the Sobolev-type inner product

$$
\langle f, g\rangle:=(f, g)+\sum_{i, j}^{p} f^{(i)}(c) a_{i j} \overline{g^{(j)}(c)},
$$

with $A=\left(a_{i j}\right)_{i, j}^{p} \quad$. If we write $\mathbf{f}(z)=\left[f(z), f^{\prime}(z), \ldots, f^{(p)}(z)\right]$, then

$$
\langle f, g\rangle=(f, g)+\mathbf{f}(c) A[\mathbf{g}(c)]^{H} .
$$

We say that $\psi_{n} \in \mathbb{P}_{n}$ is the $n$th (left) orthonormal polynomial with respect to $\langle\cdot, \cdot\rangle$ if

$$
\begin{aligned}
& \left\langle\psi_{n}(z), z^{k}\right\rangle=0 \quad(k=0,1, \ldots, n-1) \\
& \left|\left\langle\psi_{n}, \psi_{n}\right\rangle\right|=1
\end{aligned}
$$


The definition for the right orthonormal polynomials is analogous. Both of them are the same when $A$ is an Hermitian matrix, because $\langle\cdot, \cdot\rangle$ is Hermitian.

Proposition 4.1. Assume that $\psi_{n}$ exists. Then,

$$
\psi_{n}(z)=\frac{\gamma_{n}}{\kappa_{n}} \varphi_{n}(z)-\psi_{n}(c) A\left[\mathbf{K}_{n-1}(z, c)\right]^{\mathrm{T}}
$$

holds, where $\gamma_{n}>0$ is the leading coefficient of $\psi_{n}$.

Proof. Notice that $\psi_{n}(z)-\left(\gamma_{n} / \kappa_{n}\right) \varphi_{n}(z) \in \mathbb{P}_{n-1}$. Then, we have

$$
\left(\psi_{n}(y)-\frac{\gamma_{n}}{\kappa_{n}} \varphi_{n}(y), K_{n-1}(y, z)\right)=\psi_{n}(z)-\frac{\gamma_{n}}{\kappa_{n}} \varphi_{n}(z) .
$$

On the other hand,

$$
\begin{aligned}
\left(\psi_{n}(y)-\frac{\gamma_{n}}{\kappa_{n}} \varphi_{n}(y), K_{n-1}(y, z)\right) & =\left(\psi_{n}(y), K_{n-1}(y, z)\right) \\
& =\left\langle\psi_{n}(y), K_{n-1}(y, z)\right\rangle-\psi_{n}(c) A\left[\mathbf{K}_{n-1}(z, c)\right]^{\mathrm{T}} \\
& =-\psi_{n}(c) A\left[\mathbf{K}_{n-1}(z, c)\right]^{\mathrm{T}} . \quad \square
\end{aligned}
$$

Proposition 4.2. Assume that $\psi_{n}$ exists. Then,

$$
\boldsymbol{\psi}_{n}(c)[I+A M(n-1)]=\frac{\gamma_{n}}{\kappa_{n}} \boldsymbol{\varphi}_{n}(c)
$$

holds.

Proof. Taking derivatives of order $p$ in (4.1) for $z=c$,

$$
\psi_{n}(c)=\frac{\gamma_{n}}{\kappa_{n}} \boldsymbol{\varphi}_{n}(c)-\psi_{n}(c) A M(n-1) .
$$

Proposition 4.3. If $\psi_{n}$ exists, then we get

$$
\frac{\gamma_{n}}{\kappa_{n}}\left(\varphi_{n}, \varphi_{n}\right)=\frac{\kappa_{n}}{\gamma_{n}}\left\langle\psi_{n}, \psi_{n}\right\rangle-\psi_{n}(c) A\left[\boldsymbol{\varphi}_{n}(c)\right]^{H} .
$$

\section{Proof. From}

$$
\begin{aligned}
& \psi_{n}(z)=\frac{\gamma_{n}}{\kappa_{n}} \varphi_{n}(z)+(\text { lower degree terms }), \\
& \varphi_{n}(z)=\frac{\kappa_{n}}{\gamma_{n}} \psi_{n}(z)+(\text { lower degree terms }),
\end{aligned}
$$


we obtain

$$
\begin{aligned}
\left(\psi_{n}, \varphi_{n}\right) & =\frac{\gamma_{n}}{\kappa_{n}}\left(\varphi_{n}, \varphi_{n}\right), \\
\left(\psi_{n}, \varphi_{n}\right) & =\left\langle\psi_{n}, \varphi_{n}\right\rangle-\psi_{n}(c) A\left[\boldsymbol{\varphi}_{n}(c)\right]^{H} \\
& =\left\langle\psi_{n}, \frac{\kappa_{n}}{\gamma_{n}} \psi_{n}\right\rangle-\psi_{n}(c) A\left[\boldsymbol{\varphi}_{n}(c)\right]^{H} \\
& =\frac{\kappa_{n}}{\gamma_{n}}\left\langle\psi_{n}, \psi_{n}\right\rangle-\psi_{n}(c) A\left[\boldsymbol{\varphi}_{n}(c)\right]^{H} .
\end{aligned}
$$

Theorem 4.4. If $\operatorname{det}[I+A M(n-1)] \neq 0$, then the nth Sobolev-type orthonormal polynomial $\psi_{n}$ exists if and only if $\operatorname{det}[I+A M(n)] \neq 0$.

Proof. Assume that $\operatorname{det}[I+A M(n-1)] \neq 0$. Define the polynomial

$$
\Psi_{n}(z):=\varphi_{n}(z)-\boldsymbol{\varphi}_{n}(c)[I+A M(n-1)]^{-1} A\left[\mathbf{K}_{n-1}(z, c)\right]^{\mathrm{T}} .
$$

Then, if we derive $p$ times in the above expression and we evaluate it in $z=c$, we have

$$
\begin{aligned}
\boldsymbol{\Psi}_{n}(c) & =\boldsymbol{\varphi}_{n}(c)-\boldsymbol{\varphi}_{n}(c)[I+A M(n-1)]^{-1} A M(n-1) \\
& =\boldsymbol{\varphi}_{n}(c)[I+A M(n-1)]^{-1}
\end{aligned}
$$

For $k=0,1, \ldots, n-1$, we get

$$
\begin{aligned}
\left\langle\Psi_{n}, \varphi_{k}\right\rangle= & \left(\Psi_{n}, \varphi_{k}\right)+\boldsymbol{\Psi}_{n}(c) A\left[\boldsymbol{\varphi}_{k}(c)\right]^{H} \\
= & \left(\varphi_{n}, \varphi_{k}\right)-\boldsymbol{\varphi}_{n}(c)[I+A M(n-1)]^{-1} A\left[\boldsymbol{\varphi}_{k}(c)\right]^{H} \\
& +\boldsymbol{\Psi}_{n}(c) A\left[\boldsymbol{\varphi}_{k}(c)\right]^{H} .
\end{aligned}
$$

Thus, if $k<n,\left\langle\Psi_{n}, \varphi_{k}\right\rangle=0$ follows immediately. For $k=n$ we have

$$
\left\langle\Psi_{n}, \varphi_{n}\right\rangle=1+\boldsymbol{\Psi}_{n}(c) A\left[\boldsymbol{\varphi}_{n}(c)\right]^{H}=1+\boldsymbol{\varphi}_{n}(c)[I+A M(n-1)]^{-1} A\left[\boldsymbol{\varphi}_{n}(c)\right]^{H}
$$

Now, consider the matrix identities

$$
\begin{aligned}
& \left(\begin{array}{cc}
I & A\left[\boldsymbol{\varphi}_{n}(c)\right]^{H} \\
0 & 1
\end{array}\right)\left(\begin{array}{cc}
I+A M(n-1) & -A\left[\boldsymbol{\varphi}_{n}(c)\right]^{H} \\
\boldsymbol{\varphi}_{n}(c) & 1
\end{array}\right) \\
& \quad=\left(\begin{array}{cc}
I+A M(n) & 0 \\
\boldsymbol{\varphi}_{n}(c) & 1
\end{array}\right)
\end{aligned}
$$




$$
\begin{aligned}
& \left(\begin{array}{cc}
I+A M(n-1) & -A\left[\boldsymbol{\varphi}_{n}(c)\right]^{H} \\
\boldsymbol{\varphi}_{n}(c) & 1
\end{array}\right) \\
& \quad \times\left(\begin{array}{cc}
I & {[I+A M(n-1)]^{-1} A\left[\boldsymbol{\varphi}_{n}(c)\right]^{H}} \\
0 & 1
\end{array}\right) \\
& \quad=\left(\begin{array}{cc}
I+A M(n-1) & 0 \\
\boldsymbol{\varphi}_{n}(c) & 1+\boldsymbol{\varphi}_{n}(c)[I+A M(n-1)]^{-1} A\left[\boldsymbol{\varphi}_{n}(c)\right]^{H}
\end{array}\right),
\end{aligned}
$$

whose determinants are equal. Then

$$
\begin{aligned}
\operatorname{det}[I+A M(n)]=\operatorname{det}[I+A M(n-1)] & \left(1+\boldsymbol{\varphi}_{n}(c)[I+A M(n-1)]^{-1}\right. \\
& \left.\times A\left[\boldsymbol{\varphi}_{n}(c)\right]^{H}\right)
\end{aligned}
$$

and so

$$
\left\langle\Psi_{n}, \varphi_{n}\right\rangle=1+\boldsymbol{\varphi}_{n}(c)[I+A M(n-1)]^{-1} A\left[\boldsymbol{\varphi}_{n}(c)\right]^{H}=\frac{\operatorname{det}[I+A M(n)]}{\operatorname{det}[I+A M(n-1)]} .
$$

From here, the statement follows.

Besides, notice that $\psi_{n}(z)=\Psi_{n}(z) /\left|<\Psi_{n}, \Psi_{n}>\right|^{1 / 2}=\gamma_{n} z^{n}+$ (lower degree terms), with $\gamma_{n}>0$, verifies

$$
\psi_{n}(z)=\frac{\gamma_{n}}{\kappa_{n}}\left(\varphi_{n}(z)-\boldsymbol{\varphi}_{n}(c)[I+A M(n-1)]^{-1} A\left[\mathbf{K}_{n-1}(z, c)\right]^{\mathrm{T}}\right) .
$$

If $A$ is positive semidefinite, $A+[M(n-1)]^{-1}$ is positive definite $(n \geqslant p+1)$, according to Proposition 2.1. Hence, we get

$$
\operatorname{det}[I+A M(n-1)]=\operatorname{det}[M(n-1)] \times \operatorname{det}\left(A+[M(n-1)]^{-1}\right)>0
$$

and, thus

Corollary 4.5. Let $A$ be positive semidefinite. Then, the Sobolev-type orthonormal polynomial sequence exists for $n \geqslant p+1$.

Remark. From (4.2) and Proposition 4.3

$$
\left(\frac{\kappa_{n}}{\gamma_{n}}\right)^{2}=1+\boldsymbol{\Psi}_{n}(c) A\left[\boldsymbol{\varphi}_{n}(c)\right]^{H}=1+\frac{\kappa_{n}}{\gamma_{n}} \boldsymbol{\psi}_{n}(c) A\left[\boldsymbol{\varphi}_{n}(c)\right]^{H}
$$

follows.

Now, we are going to study the behavior of the $n$th Sobolev-type orthonormal polynomial $\psi_{n}$ in terms of the matrix $A$ which will be considered as a parameter. 
Theorem 4.6. Let $A$ be a positive definite matrix. Write $\sigma=1 /\left(\rho\left(A^{-1}\right)\right)$, where $\rho(M)$ is the spectral radius of $M$. Then, for fixed $n \in \mathbb{N}$,

$$
\lim _{\sigma \rightarrow \infty} \psi_{n+p+1}(z ; A)=(z-c)^{p+1} \varphi_{n}\left(z ; \mathrm{d} \mu_{p+1}\right)
$$

holds l. u. in $\mathbb{C}$.

[Notice that $\sigma \rightarrow \infty$ is equivalent to the fact that all eigenvalues of $A$ tend to $\infty$.]

Proof. From (4.4),

$$
\begin{aligned}
\psi_{n+p+1}(z ; A)= & \frac{\gamma_{n+p+1}(A)}{\kappa_{n+p+1}} \\
& \times\left(\varphi_{n+p+1}(z)-\boldsymbol{\varphi}_{n+p+1}(c)\left[A^{-1}+M(n+p)\right]^{-1}\left[\mathbf{K}_{n+p}(z, c)\right]^{\mathrm{T}}\right)
\end{aligned}
$$

holds because $A$ is nonsingular. From (4.5) and (4.2), we get

$$
\left(\frac{\kappa_{n+p+1}}{\gamma_{n+p+1}(A)}\right)^{2}=1+\boldsymbol{\varphi}_{n+p+1}(c)\left[A^{-1}+M(n+p)\right]^{-1}\left[\boldsymbol{\varphi}_{n+p+1}(c)\right]^{H} .
$$

Thus, since $M(n+p)$ is positive definite and $\lim _{\sigma \rightarrow \infty} A^{-1}=0$, it follows that

$$
\begin{aligned}
\lim _{\sigma \rightarrow \infty}\left(\frac{\kappa_{n+p+1}}{\gamma_{n+p+1}(A)}\right)^{2} & =1+\boldsymbol{\varphi}_{n+p+1}(c)[M(n+p)]^{-1}\left[\boldsymbol{\varphi}_{n+p+1}(c)\right]^{H} \\
& =\left(\frac{\kappa_{n+p+1}}{\kappa_{n}\left(\mathrm{~d} \mu_{p+1}\right)}\right)^{2} .
\end{aligned}
$$

Furthermore,

$$
\begin{aligned}
& \frac{\kappa_{n+p+1}}{\gamma_{n+p+1}(A)} \psi_{n+p+1}(z ; A)-\frac{\kappa_{n+p+1}}{\kappa_{n}\left(\mathrm{~d} \mu_{p+1}\right)}(z-c)^{p+1} \varphi_{n}\left(z ; \mathrm{d} \mu_{p+1}\right) \\
& \quad=\boldsymbol{\varphi}_{n+p+1}(c)\left([M(n+p)]^{-1}-\left[A^{-1}+M(n+p)\right]^{-1}\right)\left[\mathbf{K}_{n+p}(z, c)\right]^{\mathrm{T}} .
\end{aligned}
$$

Let $n$ be fixed, and let us consider an arbitrary compact subset $H \subset \mathbb{C}$. Then, $\left|K_{n+p}^{(0, i)}(z, c)\right|$ is uniformly bounded in $H$. For $\sigma \rightarrow \infty$, we obtain from here that

$$
\lim _{\sigma \rightarrow \infty}\left[\psi_{n+p+1}(z ; A)-(z-c)^{p+1} \varphi_{n}\left(z ; \mathrm{d} \mu_{p+1}\right)\right]=0
$$

l. u. in $\mathbb{C}$. 
Theorem 4.7. Let $\mu \in \mathscr{N}$ and $|c|>1$. If $A$ is a nonsingular matrix, then there is $n_{0} \in \mathbb{N}$ such that the (left) orthonormal polynomial $\psi_{n}$ with respect to a Sobolevtype inner product

$$
\langle f, g\rangle=\int_{|z| 1} f(z) \overline{g(z)} \mathrm{d} \mu(z)+\mathbf{f}(c) A[\mathbf{g}(c)]^{H}
$$

exists for all $n \geqslant n_{0}$.

Proof. Since Theorem 4.4, we can guarantee that there exists $\psi_{n}$, for all $n \geqslant n_{0}$, when $\operatorname{det}(I+A M(n-1)) \neq 0$. Notice that

$$
\operatorname{det}[I+A M(n-1)]=\operatorname{det}(A) \cdot \operatorname{det}[M(n-1)] \cdot \operatorname{det}\left(I+A^{-1}[M(n-1)]^{-1}\right) .
$$

As $\lim _{n} \rho\left([M(n-1)]^{-1}\right)=0$, then $\lim _{n} \operatorname{det}\left(I+A^{-1}[M(n-1)]^{-1}\right)=\operatorname{det}(I)=1$ (Theorem 3.13), that is, there exists $n_{0} \in \mathbb{N}$ such that $\operatorname{det}[I+A M(n-1)]>0$, for $n \geqslant n_{0}$.

By using (4.1), then

$$
\psi_{n}(z)=\frac{\gamma_{n}}{\kappa_{n}}\left(\varphi_{n}(z)-\boldsymbol{\varphi}_{n}(c)[I+A M(n-1)]^{-1} A\left[\mathbf{K}_{n-1}(z, c)\right]^{\mathrm{T}}\right) .
$$

If we denote

$$
\mathbf{R}_{n}(z):=[I+A M(n)]^{-1} A\left[\mathbf{K}_{n}(z, c)\right]^{\mathrm{T}},
$$

it follows that

$$
\psi_{n}(z)=\frac{\gamma_{n}}{\kappa_{n}}\left[\varphi_{n}(z)-\boldsymbol{\varphi}_{n}(c) \mathbf{R}_{n-1}(z)\right]
$$

The $\mathbf{R}_{n}(z)$ are the analogous expressions to the $\mathbf{T}_{n}(z)$, which are defined in (3.13), and those verify a similar relationship to the one given in Proposition 3.14:

Proposition 4.8. Assume that $I+A M(n+1)$ and $I+A M(n)$ are nonsingular matrices. Then,

$$
\mathbf{R}_{n+1}(z)=\mathbf{R}_{n}(z)+\frac{\kappa_{n+1}}{\gamma_{n+1}}[I+A M(n+1)]^{-1} A\left[\boldsymbol{\varphi}_{n+1}(c)\right]^{H} \psi_{n+1}(z)
$$

holds. 
Proof. The nonsingularity of $I+A M(n+1)$, and $I+A M(n)$ implies that the polynomial $\psi_{n+1}$ exists. Thus, we have

$$
\begin{aligned}
{[I+A M(n+1)]\left[\mathbf{R}_{n+1}(z)-\mathbf{R}_{n}(z)\right]=} & {[I+A M(n+1)] \mathbf{R}_{n+1}(z) } \\
& -\left[I+A M(n)+A\left[\boldsymbol{\varphi}_{n+1}(c)\right]^{H} \boldsymbol{\varphi}_{n+1}(c)\right] \mathbf{R}_{n}(z) .
\end{aligned}
$$

From here, according to (4.6),

$$
\begin{aligned}
{[I+A M(n+1)]\left[\mathbf{R}_{n+1}(z)-\mathbf{R}_{n}(z)\right]=} & A\left(\left[\mathbf{K}_{n+1}(z, c)\right]^{\mathrm{T}}-\left[\mathbf{K}_{n}(z, c)\right]^{\mathrm{T}}\right) \\
& -A\left[\boldsymbol{\varphi}_{n+1}(c)\right]^{H} \boldsymbol{\varphi}_{n+1}(c) \mathbf{R}_{n}(z) \\
= & A\left[\boldsymbol{\varphi}_{n+1}(c)\right]^{H}\left[\varphi_{n+1}(z)-\boldsymbol{\varphi}_{n+1}(c) \mathbf{R}_{n}(z)\right] \\
= & A\left[\boldsymbol{\varphi}_{n+1}(c)\right]^{H} \frac{\kappa_{n+1}}{\gamma_{n+1}} \psi_{n+1}(z) .
\end{aligned}
$$

Proposition 4.9. Assume that $\psi_{n}$ exists. Then, there exist two polynomials $P^{A}(z ; n)$, and $Q^{A}(z ; n)$, with $\operatorname{deg} P^{A}(z ; n)=p+1$ and $\operatorname{deg} Q^{A}(z ; n) \leqslant p$, such that

$$
(1-\bar{c} z)^{p+1} \psi_{n}(z)=P^{A}(z ; n) \varphi_{n}(z)+Q^{A}(z ; n) \varphi_{n}^{*}(z)
$$

holds for $c \neq 0$.

Proof. In the same way as in Proposition 3.15, we obtain

$$
\begin{aligned}
& P^{A}(z ; n)=\frac{\gamma_{n}}{\kappa_{n}}\left((1-\bar{c} z)^{p+1}+\sum_{k 0}^{p} z^{k}(1-\bar{c} z)^{p-k} \boldsymbol{\varphi}_{n}(c)\left[A^{-1}+M(n-1)\right]^{-1}\right. \\
& \left.\quad \times\left[\boldsymbol{\varphi}_{n}(c) B^{k}\right]^{H}\right) \\
& Q^{A}(z ; n)=-\frac{\gamma_{n}}{\kappa_{n}} \sum_{k 0}^{p} z^{k}(1-\bar{c} z)^{p-k} \boldsymbol{\varphi}_{n}(c)\left[A^{-1}+M(n-1)\right]^{-1}\left[\boldsymbol{\varphi}_{n}^{*}(c) B^{k}\right]^{H} .
\end{aligned}
$$

\section{Asymptotic behavior for $\psi_{n}$}

We will assume that $\mu \in \mathscr{N},|c|>1$ and $A$ is a nonsingular matrix. In these conditions, Theorems 3.13 and 4.7 hold, and the existence for the $n$th Sobolevtype orthonormal polynomial $\psi_{n}=\gamma_{n} z^{n}+$ (lower degree terms) for $n$ large enough is guaranteed.

Proposition 5.1. $\lim _{n} \kappa_{n} / \gamma_{n}=|c|^{p+1}$. 
Proof. From (4.5), we get

$$
\begin{aligned}
\left(\frac{\kappa_{n}}{\gamma_{n}}\right)^{2} & =\frac{\operatorname{det}[I+A M(n)]}{\operatorname{det}[I+A M(n-1)]} \\
& =\frac{\operatorname{det}[M(n)]}{\operatorname{det}[M(n-1)]} \cdot \frac{\operatorname{det}\left[I+A^{-1}[M(n)]^{-1}\right]}{\operatorname{det}\left(I+A^{-1}[M(n-1)]^{-1}\right)} .
\end{aligned}
$$

Now, by using Theorem 3.13, we obtain

$$
\lim _{n} \operatorname{det}\left(I+A^{-1}[M(n)]^{-1}\right)=1
$$

and, thus, we conclude that

$$
\begin{aligned}
\lim _{n}\left(\frac{\kappa_{n}}{\gamma_{n}}\right)^{2} & =\lim _{n} \frac{\operatorname{det}[M(n)]}{\operatorname{det}[M(n-1)]}=\lim _{n}\left(1+\boldsymbol{\varphi}_{n}(c)[M(n-1)]^{-1}\left[\boldsymbol{\varphi}_{n}(c)\right]^{H}\right) \\
& =|c|^{2 p+2}
\end{aligned}
$$

according to (3.2) and Corollary 3.7.

Theorem 5.2. $\lim _{n} \psi_{n}(z) / \varphi_{n}(z)=(\bar{c} /|c| \cdot(z-c) / \bar{c} z-1)^{p+1}$ uniformly in $|z| \geqslant 1$, or, equivalently, $\lim _{n} \psi_{n}(z) /\left(\varphi_{n-p-1}\left(z ; \mathrm{d} \mu_{p+1}\right)\right)=(z-c)^{p+1}$ uniformly in $|z| \geqslant 1$.

Proof. Note that the equivalence for these both conditions follows immediately from Proposition 3.6.

We shall denote $\hat{\varphi}_{n}(z)=\left(1 / \kappa_{n}\right) \varphi_{n}(z), \quad \hat{\varphi}_{n}\left(z ; \mathrm{d} \mu_{p+1}\right)=\left(1 / \kappa_{n}\left(\mathrm{~d} \mu_{p+1}\right)\right) \varphi_{n}$ $\left(z ; \mathrm{d} \mu_{p+1}\right)$, and $\hat{\psi}_{n}(z)=\left(1 / \gamma_{n}\right) \psi_{n}(z)$ the corresponding $n$th monic orthogonal polynomials. From (3.14) and (4.7), we have

$$
\begin{aligned}
\frac{(z-c)^{p+1} \hat{\varphi}_{n-p-1}\left(z ; \mathrm{d} \mu_{p+1}\right)}{\hat{\varphi}_{n}(z)}-\frac{\hat{\psi}_{n}(z)}{\hat{\varphi}_{n}(z)} & =\frac{\boldsymbol{\varphi}_{n}(c)\left[\mathbf{R}_{n-1}(z)-\mathbf{T}_{n-1}(z)\right]}{\hat{\varphi}_{n}(z)} \\
& =\frac{\boldsymbol{\varphi}_{n}(c)\left[\mathbf{R}_{n-1}(z)-\mathbf{T}_{n-1}(z)\right]}{\varphi_{n}(z)} .
\end{aligned}
$$

We prove that

$$
\lim _{n}\left[\boldsymbol{\varphi}_{n}(c) \cdot \frac{\mathbf{T}_{n-1}(z)-\mathbf{R}_{n-1}(z)}{\varphi_{n}(z)}\right]=0
$$

uniformly in $|z| \geqslant 1$.

As $A$ is nonsingular, we get

$$
\mathbf{R}_{n-1}(z)=\left(I+[M(n-1)]^{-1} A^{-1}\right)^{-1} \mathbf{T}_{n-1}(z),
$$


according to (3.13) and (4.6). Hence,

$$
\begin{aligned}
\boldsymbol{\varphi}_{n}(c) \cdot \frac{\mathbf{T}_{n-1}(z)-\mathbf{R}_{n-1}(z)}{\varphi_{n}(z)}= & \boldsymbol{\varphi}_{n}(c)\left[I-\left(I+[M(n-1)]^{-1} A^{-1}\right)^{-1}\right] \frac{\mathbf{T}_{n-1}(z)}{\varphi_{n}(z)} \\
= & \boldsymbol{\varphi}_{n}(c)[M(n-1)]^{-1} A^{-1}\left(I+[M(n-1)]^{-1} A^{-1}\right)^{-1} \\
& \times \frac{\mathbf{T}_{n-1}(z)}{\varphi_{n}(z)}
\end{aligned}
$$

holds and, thus,

$$
\begin{aligned}
\left|\boldsymbol{\varphi}_{n}(c) \cdot \frac{\mathbf{T}_{n-1}(z)-\mathbf{R}_{n-1}(z)}{\varphi_{n}(z)}\right| \leqslant & \left\|\boldsymbol{\varphi}_{n}(c)\right\| \cdot \rho\left([M(n-1)]^{-1}\right) \cdot\left\|A^{-1}\right\| \\
& \cdot\left\|\left(I+[M(n-1)]^{-1} A^{-1}\right)^{-1}\right\| \cdot \mid \frac{\left\|\mathbf{T}_{n-1}(z)\right\|}{\left|\varphi_{n}(z)\right|} .
\end{aligned}
$$

Here, the matrix norm $\|C\|=\sqrt{ } \rho\left(C C^{H}\right)$ is used. Because $\rho\left([M(n)]^{-1}\right)$ tends to zero, then $\lim _{n}\left\|\left(I+[M(n-1)]^{-1} A^{-1}\right)^{-1}\right\|=1$. Thus, to prove (5.1) is equivalent to prove

$$
\lim _{n}\left\|\boldsymbol{\varphi}_{n}(c)\right\| \rho\left([M(n-1)]^{-1}\right) \frac{\left\|\mathbf{T}_{n-1}(z)\right\|}{\left|\varphi_{n}(z)\right|}=0
$$

uniformly in $|z| \geqslant 1$.

First, from (3.11) (Theorem 3.13), we get

$$
\rho\left([M(n-1)]^{-1}\right)=\mathcal{O}\left(\frac{\left|\prod_{k 0}^{p} \varphi_{n}^{(p-k)}(c)\right|^{2}}{\left|\varphi_{n}(c)\right|^{2(p+2)}}\right),
$$

and thus

$$
\begin{aligned}
\left\|\boldsymbol{\varphi}_{n}(c)\right\| \rho\left([M(n-1)]^{-1}\right) & =\mathcal{O}\left(\left|\varphi_{n}^{(p)}(c)\right|\right) \cdot \mathcal{O}\left(\frac{\left|\prod_{k}^{p} \varphi_{n}^{(p-k)}(c)\right|^{2}}{\left|\varphi_{n}(c)\right|^{2(p+2)}}\right) \\
& =\mathcal{O}\left(\left|\frac{\varphi_{n}^{(p)}(c)}{\left[\varphi_{n}(c)\right]^{1+r}}\right|\right) \cdot \mathcal{O}\left(\prod_{k}^{p}\left|\frac{\varphi_{n}^{(p-k)}(c)}{\left[\varphi_{n}(c)\right]^{1+r}}\right|^{2}\right)
\end{aligned}
$$

follows, with $r=1 /(2 p+3)>0$. Hence $\lim _{n}\left\|\boldsymbol{\varphi}_{n}(c)\right\| \rho\left([M(n-1)]^{-1}\right)=0$ (Lemma 3.4). 
Furthermore, by considering (3.13) and (3.15) we can obtain the following upper bound

$$
\begin{aligned}
\frac{\left\|\mathbf{T}_{n-1}(z)\right\|}{\left|\varphi_{n}(z)\right|} & \leqslant \frac{\rho\left([M(n-1)]^{-1}\right)|| \mathbf{K}_{n-1}(z, c) \|}{\left|\varphi_{n}(z)\right|} \\
& \leqslant \frac{\rho\left([M(n-1)]^{-1}\right)}{|1-\bar{c} z|} \cdot\left\|\frac{\overline{\varphi_{n}^{*}(z)}}{\overline{\varphi_{n}(z)}} \boldsymbol{\varphi}_{n}^{*}(c)-\boldsymbol{\varphi}_{n}(c)\right\| \cdot \sum_{k=0}^{p}\left|\frac{z}{1-\bar{c} z}\right|^{k}\|B\|^{k} .
\end{aligned}
$$

Now,

$$
\begin{aligned}
& \rho\left([M(n-1)]^{-1}\right)\left\|\overline{\frac{\overline{\varphi_{n}^{*}(z)}}{\bar{\varphi}_{n}(z)}} \boldsymbol{\varphi}_{n}^{*}(c)-\boldsymbol{\varphi}_{n}(c)\right\| \\
& \quad \leqslant \rho\left([M(n-1)]^{-1}\right) \cdot\left\|\boldsymbol{\varphi}_{n}(c)\right\|\left(1+\left|\frac{\varphi_{n}^{*}(z)}{\varphi_{n}(z)}\right| \frac{\left\|\boldsymbol{\varphi}_{n}^{*}(c)\right\|}{\left\|\boldsymbol{\varphi}_{n}(c)\right\|}\right),
\end{aligned}
$$

with

$$
\left|\frac{\varphi_{n}^{*}(z)}{\varphi_{n}(z)}\right| \frac{\left\|\varphi_{n}^{*}(c)\right\|}{\left\|\varphi_{n}(c)\right\|} \leqslant\left|\frac{\varphi_{n}^{*}(z)}{\varphi_{n}(z)}\right|\left(\sum_{k 0}^{p}\left|\frac{\varphi_{n}^{*(k)}(c)}{\varphi_{n}^{(k)}(c)}\right|^{2}\right)^{1 / 2}
$$

where the first factor is uniformly bounded by 1 in $|z| \geqslant 1$, and the second one tends to zero (Lemma 2.4 and (2.2)). Also, $z /(1-\bar{c} z)$ and $1 /(1-\bar{c} z)$ are uniformly bounded by $1 /(|c|-1)$ in $|z| \geqslant 1$.

In these conditions,

$$
\lim _{n} \frac{\left\|\mathbf{T}_{n-1}(z)\right\|}{\left|\varphi_{n}(z)\right|} \leqslant \sum_{k 0}^{p} \frac{\|B\|^{k}}{(|c|-1)^{k+1}} \cdot \lim _{n}\left[\rho\left([M(n-1)]^{-1}\right)\left\|\boldsymbol{\varphi}_{n}(c)\right\|\right]=0 .
$$

follows immediately.

Hence

$$
\lim _{n} \frac{\hat{\psi}_{n}(z)}{\hat{\varphi}_{n}(z)}=\lim _{n} \frac{(z-c)^{p+1} \hat{\varphi}_{n-p-1}\left(z ; \mathrm{d} \mu_{p+1}\right)}{\hat{\varphi}_{n}(z)},
$$

i.e.,

$$
\lim _{n} \frac{\kappa_{n}}{\gamma_{n}} \frac{\psi_{n}(z)}{\varphi_{n}(z)}=\lim _{n} \frac{\kappa_{n}}{\kappa_{n-p-1}\left(\mathrm{~d} \mu_{p+1}\right)} \frac{(z-c)^{p+1} \varphi_{n-p-1}\left(z ; \mathrm{d} \mu_{p+1}\right)}{\varphi_{n}(z)} .
$$


But, Propositions 3.6 and 5.1 yield

$$
\lim _{n} \frac{\kappa_{n}}{\gamma_{n}}=\lim _{n} \frac{\kappa_{n}}{\kappa_{n-p-1}\left(\mathrm{~d} \mu_{p+1}\right)} .
$$

So, the statement is proved.

Corollary 5.3. There is $n_{0} \in \mathbb{N}$ such that, for $n \geqslant n_{0}$, the nth Sobolev-type orthonormal polynomial $\psi_{n}$ has exactly $p+1$ zeros in $|z|>1$, which accumulate in $c$, while the remaining zeros belong to $|z|<1$.

Proof. Taking into account that

$$
\lim _{n} \frac{\psi_{n}(z)}{\varphi_{n-p-1}\left(z ; \mathrm{d} \mu_{p+1}\right)}=(z-c)^{p+1}
$$

uniformly in $|z| \geqslant 1$, the result follows immediately from Hurwitz's Theorem.

Theorem 5.4. For the polynomial coefficients $P^{A}(z ; n)$ and $Q^{A}(z ; n)$ in Proposition 4.9 ,

$$
\lim _{n} P^{A}(z ; n)=\left[\frac{\bar{c}}{|c|}(c-z)\right]^{p+1}, \quad \lim _{n} Q^{A}(z ; n)=0
$$

hold l. u. in $\mathbb{C}$.

Proof. Consider the expressions in Propositions 3.15 and 4.9. Then, we can write

$$
\begin{aligned}
\frac{\kappa_{n}}{\kappa_{n-p-1}\left(\mathrm{~d} \mu_{p+1}\right)} P(z ; n)-\frac{\kappa_{n}}{\gamma_{n}} P^{A}(z ; n)= & \sum_{k 0}^{p} z^{k}(1-\bar{c} z)^{p-k} \boldsymbol{\varphi}_{n}(c)\left([M(n-1)]^{-1}\right. \\
& \left.-\left[A^{-1}+M(n-1)\right]^{-1}\right)\left[\boldsymbol{\varphi}_{n}(c) B^{k}\right]^{H} .
\end{aligned}
$$

But, from

$$
\begin{aligned}
& {[M(n-1)]^{-1}-\left[A^{-1}+M(n-1)\right]^{-1}} \\
& \quad=[M(n-1)]^{-1}\left(I+A^{-1}[M(n-1)]^{-1}\right)^{-1} A^{-1}[M(n-1)]^{-1},
\end{aligned}
$$

it follows that

$$
\begin{aligned}
& \frac{\kappa_{n}}{\kappa_{n-p-1}\left(\mathrm{~d} \mu_{p+1}\right)}\left|P(z ; n)-\frac{\kappa_{n-p-1}\left(\mathrm{~d} \mu_{p+1}\right)}{\gamma_{n}} P^{A}(z ; n)\right| \\
& \leqslant\left\|\boldsymbol{\varphi}_{n}(c)\right\|^{2}\left[\rho\left([M(n-1)]^{-1}\right)\right]^{2} \\
& \quad \times\left.\left\|\left(I+A^{-1}[M(n-1)]^{-1}\right)^{-1}\right\|||\left|A^{-1}\right|\left|\sum_{k 0}^{p}\right| z\right|^{k}|1-\bar{c} z|^{p-k}|| B^{k} \| .
\end{aligned}
$$


In Theorem 5.2, $\lim _{n}\left\|\left(I+A^{-1}[M(n-1)]^{-1}\right)^{-1}\right\|=1$ and $\lim _{n}\left\|\boldsymbol{\varphi}_{n}(c)\right\|$ $\rho\left([M(n-1)]^{-1}\right)=0$ are stated. Besides, we have

$$
\lim _{n} \frac{\kappa_{n-p-1}\left(\mathrm{~d} \mu_{p+1}\right)}{\gamma_{n}}=\lim _{n} \frac{\kappa_{n-p-1}\left(\mathrm{~d} \mu_{p+1}\right)}{\kappa_{n}} \cdot \lim _{n} \frac{\kappa_{n}}{\gamma_{n}}=1
$$

according to Propositions 5.1 and 3.6. Thus, Theorem 3.16 implies the statement for $P^{A}(z ; n)$.

The proof is similar for $Q^{A}(z ; n)$.

\section{Acknowledgements}

The work of the first author (F. Marcellán) was partially supported by D.G.E.S. of Spain under grant PB96-0120-C03-01. The work of the second author (L. Moral) was partially supported by P.A.I. 1997 (Universidad de Zaragoza) CIE-10. The authors are grateful to the referee for the useful remarks and suggestions.

\section{References}

[1] A. Cachafeiro, F. Marcellán, Orthogonal polynomials of Sobolev type on the unit circle, J. Approx. Theory 78 (1994) 127146.

[2] A. Foulquié, F. Marcellán, K. Pan, Asymptotic behavior of Sobolev type orthogonal polynomials on the unit circle, J. Approx. Theory 100 (1999) 345363.

[3] G. Freud, Orthogonal Polynomials, Pergamon Press, Oxford, 1971.

[4] L. Ya, Geronimus, Polynomials orthogonal on a circle and their applications, Am. Math. Soc. Transl. 3 (1) (1962) 178.

[5] E. Godoy, F. Marcellán, An analog of the Christoffel formula for polynomial modification of a measure on the unit circle, Boll. Un. Mat. Ital. A 5 (7) (1991) 112.

[6] V. Grenander, G. Szego, Toeplitz Forms and their Applications, 2nd ed, Chelsea, New York, 1984.

[7] X. Li, F. Marcellán, On polynomials orthogonal with respect to a Sobolev inner product on the unit circle, Pacific J. Math. 175 (1) (1996) 127146.

[8] X. Li, F. Marcellán, Representations of orthogonal polynomials for modified measures, Comm. Anal. Theory Cont. Fractions 7 (1999) 922.

[9] G. López, F. Marcellán, W. Van Assche, Relative Asymptotics for polynomials orthogonal with respect to a discrete Sobolev inner product, Constr. Approx. 11 (1995) 107137.

[10] F. Marcellán, W. Van Assche, Relative asymptotics for orthogonal polynomials with a Sobolev inner product, J. Approx. Theory 72 (1992) 192209.

[11] A. Maté, P. Nevai, Remarks on E.A. Rahmanov's paper on the asymptotics of the ratio of orthogonal polynomials, J. Approx. Theory 36 (1982) 6472.

[12] A. Maté, P. Nevai, V. Totik, Extensions of Szego's theory of orthogonal polynomials II, Constr. Approx. 3 (1987) 5172. 
[13] O. Njastad, W.B. Jones, W. Thron, Moment theory, orthogonal polynomials, quadrature and continued fractions associated with the unit circle, Bull. London Math. Soc. 21 (1989) 113152.

[14] G. Szego, Orthogonal Polynomials, vol. 24, fourth ed., Am. Math. Soc. Coll. Publ., Amer. Math. Soc. Providence, R.I., 1975. 